\title{
Genome-wide identification and expression analysis of the VQ gene family in $C$. pepo
}

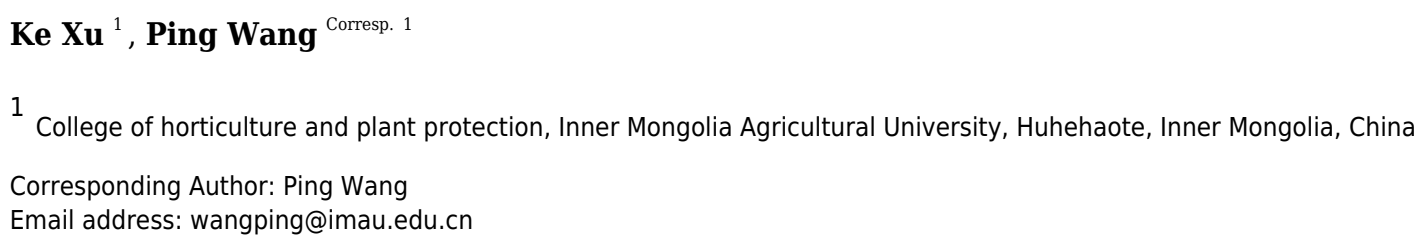

VQ protein is a plant specific protein, which plays an important role in plant growth and development and biological and abiotic stress response.This study aimed to systematically analyze for the first time the $V Q$ of $C$. pepo and understand their expression patterns in response to different stimuli. Herein, $44 V Q$ genes were identified, which were divided into eight groups (I-VIII) based on phylogenetic analysis. Two genes (CpVQ1 and CpVQ2) could not be located on the chromosome, whereas the remaining CpVQ genes were randomly distributed on the chromosomes, except for chromosomes 15 and 18. Noteworthy, the main event driving the expansion of the $V Q$ gene family was chromosome fragment duplication. Based on qRT-PCR analysis, VQ genes are expressed in different tissues, and $V Q$ genes are differentially regulated under a variety of abiotic stresses and powdery mildew stress, indicating that they play an important role in plant stress response and other aspects. This report presents the first systematic analysis of VQ genes from $C$. pepo and provides a solid foundation for further research of the specific functions of VQ proteins. 
1 Genome-wide identification and expression analysis of

2 the $V Q$ gene family in C. pepo

3

4

5

6

7

8

9

10

11

12

13

14

15

16

17

18

19

20

21

22

23

24

25

26

27

28

29

30

31

32

33

34

35

36

37

38

39

Xu Ke ${ }^{1}$, Wang Ping ${ }^{1}$

${ }^{1}$ College of horticulture and plant protection, Inner Mongolia Agricultural University, Huhehaote, Inner Mongolia, China

Corresponding Author:

Wang Ping ${ }^{1}$

University of east, Huhehaote, Inner Mongolia, 01000, China

Email address: wangping@imau.edu.cn

\section{Abstract}

VQ protein is a plant specific protein, which plays an important role in plant growth and development and biological and abiotic stress response.This study aimed to systematically analyze for the first time the $V Q$ of $C$. pepo and understand their expression patterns in response to different stimuli. Herein, $44 V Q$ genes were identified, which were divided into eight groups (I-VIII) based on phylogenetic analysis. Two genes ( $C p V Q 1$ and $C p V Q 2)$ could not be located on the chromosome, whereas the remaining $C p V Q$ genes were randomly distributed on the chromosomes, except for chromosomes 15 and 18. Noteworthy, the main event driving the expansion of the $V Q$ gene family was chromosome fragment duplication. Based on qRT-PCR analysis, $V Q$ genes are expressed in different tissues, and $V Q$ genes are differentially regulated under a variety of abiotic stresses and powdery mildew stress, indicating that they play an important role in plant stress response and other aspects. This report presents the first systematic analysis of $V Q$ genes from $C$. pepo and provides a solid foundation for further research of the specific functions of VQ proteins.

Keywords: $V Q$ genes family ; C. pepo ; expression pattern ; powdery mildew

\section{Introduction}

VQ protein is a highly conserved transcription factor that is widely expressed in biology, including several monocotyledonous and dicotyledonous plants(Jing and Lin, 2015; Song et al.,2016;Ding et al.,2019). This protein owns its name to its VQ motifs, which allows it to interact with WRKY transcription factors to regulate plant growth and development(Lai et al., 2011). For example, VQ10 interacts with WRKY25 and WRKY33, which when simultaneously overexpressed can impair plant growth(Cheng et al., 2012). In Arabidopsis, AtWRKY2/34 and $A t V Q 20$ form a complex that negatively regulates the expression of MYB transcription factors to

Peer) reviewing PDF | (2021:09:65295:2:0:NEW 27 Dec 2021) 
40 regulate the development and function of pollen(Lei et al., 2017; 2018). The ATVQ8 gene affects

41 leaf development, making the leaves appear yellow-green throughout the growth period, causing 42 plant malnutrition and affecting the normal growth of Arabidopsis plants. Plants overexpressing

$43 V Q 17, V Q 18$, and $V Q 22$ have slow growth and yellow leaves, and the leaf area becomes 44 smaller(Cheng at al., 2012). Moreover, IKU1 encodes a protein containing a VQ motif that is 45 involved in the regulation of endosperm development, which in turn affects seed size(Wang et 46 al., 2010).

47
VQ plays an important protective role on plants, allowing them to fight pathogenic bacteria and other biological and abiotic stresses(Jing et al.,2018). For example, OsVQ13 is highly expressed at $6 \mathrm{~h}$ after bacterial blight infection; thus, it may be involved in the resistance pathway of Oryza sativa to bacterial blight(Kim et al., 2013a). Some $P b r V Q$ genes are differentially expressed under gibberellin and black spot stress(Cao et al.,2018). Furthermore, transgenic Arabidopsis overexpressing AtVQ15 is highly sensitive to salt stress during seed germination and seedling growth, whereas mutants of this gene show strong salt tolerance(Perruc at al., 2004). In response to harsh environments, plants have evolved several complex mechanisms to deal with external stimuli(Fujita et al., 2006). This response is caused by the interaction of transcription factors and cofactors to coordinate the transcription mechanisms of the plant in response to the surrounding environment(Wray et al., 2003).For example, AtVQ9 acts antagonistically with AtWRKY8 to mediate responses to salt stress(Hu et al., 2013). Studies have shown that AtVQ10 and WRKY8 form a complex in the nucleus and positively regulate the resistance of Arabidopsis to Botrytis cinerea(Chen et al., 2018). The banana fruit MaWRKY26 transcription factor physically interacts with the VQ motif-containing protein MaVQ5, which leads to attenuated MaWRKY26-induced transactivation of jasmonic acid biosynthetic genes that are associated with various stress responses(Ye et al.,2016).

C. pepo belongs to the Cucurbita genus of the Cucurbitaceae family. Because it contains several nutrients, it has high edible and medicinal value, and is widely cultivated worldwide. During the cultivation process, C. pepo is susceptible to various biological and abiotic stresses that will affect the yield and quality of the fruit. Therefore, identification of resistance genes has great significance for improving the yield and quality of $C$. pepo through molecular breeding. Currently, 34, 39, 75, and $61 V Q$ genes have been identified in Arabidopsis, O. sativa, Glycine max and Maize, respectively(Cheng at al., 2012; Kim at al., 2013a; Wang et al., 2019; Song et al.,2016). However, there is no detailed description of the $V Q$ genes of C. pepo. In this study, we investigated the $V Q$ genes in the C. pepo genome, and analyzed their phylogeny, gene structure, chromosomal location, and collinearity for a comprehensive analysis. In addition, we also analyzed the expression levels of $C p V Q s$ under different abiotic stresses and powdery mildew stress. This study is expected to provide basic information for the identification and classification of $C p V Q$ genes. Further experimental analysis can allow us to understand the functions of $C p V Q$ s involved in plant stress response.

\section{Materials \& Methods}

Identification of $V Q$ genes in C. pepo

Peer) reviewing PDF | (2021:09:65295:2:0:NEW 27 Dec 2021) 
80 In present study, two methods were used to identify complete VQ members in C. pepo. Firstly, 81 The Hidden Markov Model (HMM) profiles of the VQ motif (PF05678) was downloaded from 82 PFAM protein family database (http://pfam.xfam.org/)(Punta et al.,2004). Then the model as a 83 probe to perform a BLASTp against the C. pepo genome database using HMMER 3.0. The cut84 off E-value was set as 0.1. Secondly, The known VQ motif-containing members of $A$. thaliana 85 were obtained from The Arabidopsis Information Resource (TAIR) and database 86 (http://arabidopsis.org) were used as queries to conduct a local BLASTp against the protein 87 database of $C$. pepo. The targeting genes with similarity of E-value less than $1 \mathrm{e}^{-20}$ were retained 88 for the further analysis. We set e as two values of 0.1 and $1 \mathrm{e}^{-20}$ for filtering. We set E-value to 0.1 for the initial filtering, and then set E-value to $1 \mathrm{e}^{-20}$ for in-depth filtering. After merging the two results, the candidate $V Q$ genes were evaluated using the online program PFAM(http://pfam.xfam.org/search),Conserved Domains DataBase Search (https://www.ncbi.nlm.nih.gov/Structure/cdd/wrpsb.cgi) and SMART tool (http://smart.emblheidelberg.de/) to confirm the presence of VQ motif. The biophysical properties of VQ members, such as, peptide length, molecular weight (MW), isoelectric point (pI) were predicted using the online ExPasy program (http://www.expasy.org/tools/)(Wilkins et al.,1999).

\section{Multiple sequence alignment and Phylogenetic Analysis}

In order to investigate the evolutionary relationships and classification of VQ members in $C$. pepo, we firstly conducted a multiple sequence alignment based on the full-length amino acid sequence of all $C p V Q s$ using the ClustalW tool with auto strategy parameters. According to the alignment results, we constructed a phylogenetic tree using the neighbor-joining (NJ) method with MEGAX software（Tamura et al.,2011）.Branch support for the tree topology was estimated by using a bootstrap analysis with 1000 replicates. The phylogenetic tree was illustrated using the online ITOL (http://itol.embl.de/help.cgi) online tool.

\section{Gene structure analysis and conserved motif detection}

105

106

107

108

109

110

111

112

113

114

115

116

117

118

119

The online program Gene Structure Display Server (GSDS) (http://gsds.cbi.pku.edu.cn/) was used to draw a diagrammatic sketch of the intron-exon structure by comparing their coding sequence (CDSs) with their corresponding genomic DNA sequences. The conserved motifs of $C p V Q s$ were detected by the online tool MEME (http://meme.ebi.edu.au/). The parameters were set as follows : zero or one occurrence per sequence; maximum number of motifs: 20; and other optional parameters was set default(Bailey et al.,2015).

\section{Chromosome Locations, Gene duplication and collinearity analysis}

For the further investigation of evolution in $C p V Q s$, we firstly constructed the distribution map of VQ members in C. pepo. The physical positions of all $V Q$ genes were retrieved from the GFF3 annotation file using a local script and the schematic diagram of chromosomal location was visualized by TBTOOLS(Chen et al.,2020). For the identification of tandem duplication events and segmental duplication events in $\mathrm{CpVQ}$ family, all $C p V Q s$ amino acid sequences were aligned using BLASTp, with an e-value of $1 \mathrm{e}^{-10}$. As previous research described, two or more genes located on the same chromosome were arranged in a $200 \mathrm{~kb}$ distance and shared more than $70 \%$ identity as analyzed with BLASTp can be defined as tandem duplication events. The

Peer] reviewing PDF | (2021:09:65295:2:0:NEW 27 Dec 2021) 
120 segmental duplication events were identified by using the Multiple collinear scanning toolkits 121 (MCScanX) with default parameters(Wang et al.,2012a). The Circos program was used to draw 122 collinearity maps to exhibit duplicated gene pairs between $C p V Q$ genes as well as the synteny 123 blocks of the orthologous VQ genes between C. pepo and Arabidopsis thaliana, Cucumis melo, 124 Oryza sativa, Zea mays.

\section{Calculating $\mathrm{Ka}$ and $\mathrm{Ks}$}

126

127

128

129

130

131

132

133

134

135

136

137

138

139

140

141

142

143

144

145

146

147

148

149

150

151

152

153

154

155

156

157

158

159

The Ka and Ks were calculated to assess the selection history and divergence time of gene families. The values of synonymous (Ks) and nonsynonymous (Ka) substitutions of duplicated $V Q$ genes were estimated by using the KaKs_Calculator 2.0 with the NG method(Wang et al.,2010a). The divergence time $(\mathrm{T})$ was calculated using the formula $\mathrm{T}=\mathrm{Ks} /\left(2 \times 6.1 \times 10^{-9}\right) \times 10^{-6}$ million years ago (MYA)(Kim et al., 2013a; Kim et al., 2013b).

\section{$V Q$ gene expression analysis of C. pepo}

The transcriptome expression data of $V Q$ genes under Podosphaera xanthii stress was available from our laboratory. The transcript abundance was represented by fragments per kilobase of exon per million mapped reads (FPKM) values which were calculated based on RNA-Seq reads. The heatmaps showing expression profiles were generated using log10-transformed FPKM values. The results were presented as heatmaps using TBTOOLS software(Chen et al.,2020).

\section{Plant materials and treatments}

C. pepo (Self-delivered F2) was used in present study. Seeds were planted in a 1:1 (w/w) mixture of soil and sand, cultured in an artificial climatic chamber kept at $30 / 22{ }^{\circ} \mathrm{C}$ with a $18 / 6 \mathrm{~h}$ photoperiod (day/night). Seedlings that germinated after 8 weeks were subjected to different stress conditions: $200 \mathrm{mM} \mathrm{NaCl}$ solution, $20 \%$ PEG6000 (drought), $4{ }^{\circ} \mathrm{C}$ (cold), immersing (waterlogging) and cultured for a total of $24 \mathrm{~h}$, leaves were collected after 0,12 and $24 \mathrm{~h}$. In order to analyze the expression of $V Q$ genes in different tissues, we collected plant roots, stems, and leaves for RNA preparation. All of the samples were immediately frozen in liquid nitrogen and stored at $-80^{\circ} \mathrm{C}$ for subsequent total RNA extraction. All samples were tested with three technical replicates and three independent biological replicates.

\section{RNA extraction and Quantitative real-time PCR (qRT-PCR)}

\section{Total RNA was extracted from C.peop using RNAsimple Total RNA Kit (TIANGEN} BIOTECH, Beijing) according to the manufacturer's instructions. First-strand cDNA synthesis was accomplished using TransScript One-Step gDNA Removal and cDNA Synthesis SuperMix (Transgen Biotech, Beijing). Quantitative Real-time PCR (qRT-PCR) was performed using TB Green Premix Ex Taq II (TliRNaseH Plus) (RR420Q TaKaRa Biotechnology, Beijing, China) on an FTC-3000P system (Applied Biosystems, CA, Canad) with the primers listed in Table S1. The reaction procedure was completed under the following program: $30 \mathrm{~s}$ of pre-denaturation at $95^{\circ} \mathrm{C}, 40$ cycles of $5 \mathrm{~s}$ at $95^{\circ} \mathrm{C}$, and $30 \mathrm{~s}$ at $60^{\circ} \mathrm{C}$, and $4{ }^{\circ} \mathrm{C}$ to finish. All samples were tested with three technical replicates and three independent biological replicates. The relative expression level was calculated while using the $2^{-\Delta \Delta \mathrm{CT}}$ method(Livak et al.,2001). The actin and $C A C$ genes were used as internal control.(Obrero et al.,2011).

\section{Results}




\section{Identification and sequence analysis of $V Q$ genes in C. pepo}

161 After manually removing redundant entries through screening(manually delete the genes without

162

163

164

165

166

167

168

169

170

171

172

173

174

175

176

177

178

179

180

181

182

183

184

185

186

187

188

189

190

191

192

193

194

195

196

197

198

199

VQ motif) and validation of the search results, a total of $44 V Q$ genes were identified within the whole genome of $C$. pepo, which were named $C p V Q 1-C p V Q 44$ based on their physical locations on the chromosomes. The characteristics concerning each gene, including gene number, length of coding sequence and amino acid sequence, MW and PI of the proteins, are summarized in Table 1. Subsequent sequence analysis of these $44 C p V Q$ s showed that the encoded CpVQ proteins ranged from 73 amino acids(aa) (CpVQ34) to 628 amino acids (CpVQ14) in length (average length: 219 amino acids). Similar to previous studies in Arabidopsis and O. sativa, most VQ proteins contained less than 300 amino acids. The calculated molecular weight and isoelectric points of these proteins varied from $8.34 \mathrm{kDa}(C p V Q 34)$ to $67.83 \mathrm{kDa}(C p V Q 14)$, and 4.68 ( $C p V Q 40)$ to 10.67 (CpVQ19), respectively. The length of the coding sequences of this gene family was between 222 and $1,887 \mathrm{bp}$ (average length: $660 \mathrm{bp}$ ).

\section{Phylogenetic analysis of CpVQs}

To detect the evolutionary relationships and classification of the VQ family in C. pepo, unrooted phylogenetic Neighbor-Joining trees were constructed with the $44 \mathrm{CpVQ}$ proteins and the known VQ protein from Arabidopsis. Through the analysis of the phylogenetic and structural features of the VQ domains, these proteins were divided into 8 clades(I-VIII) based on the nomenclature of the Arabidopsis VQs, with two proteins in I and VIII groups, three each in VI and VII, six members in III, eight in II, and group IV and V have the biggest amount of proteins with $10 \mathrm{VQs}$ (Fig. 1). When compared with the other groups, the size of group IV and V were significantly larger. These results were not completely consistent with previous studies in A. thaliana, $O$. sativa, and Zea mays. In addition, a phylogenic tree was built using the $44 \mathrm{CpVQ}$ protein sequences, which indicated that the groups IV and V in C. pepo had more VQ members than those in Arabidopsis. In addition, it is also possible that the expansion of the two subgroups may have resulted from gene duplications.

\section{Gene structure and conserved motifs analysis}

Gene structure can provide more information about the evolutionary relationship in a gene family; thus, the organization of exons and introns in $C p V Q s$ was investigated using the GSDS2.0 online tool. Structural analysis of $V Q s$ showed that half of the members of group V had introns, such as $C p V Q 14$ that had four introns, which may be related to their long sequences; genes in group VII have longer coding regions; whereas genes of group I have shorter coding regions than other groups. Moreover, $75 \%$ of $C p V Q s$ were found to be intronless genes, suggesting that many introns were lost during the long evolution. To further analyze the characteristics of $C p V Q s$, the MEME online tool was used to predict the potential conserved motifs. A total of 20 motifs were identified, with lengths ranging between nine and 48 amino acids. Noteworthy, every $C p V Q$ contained 1-6 conserved motifs (Fig. 2). Motif 1 contained the domain present in every VQ member; thus, representing an essential element in the VQ family. In addition, every group was found to have a clearly identifiable motif structure that distinguished it from the other groups. For example, Motif 2 and Motif 5 were prominent in

Peer) reviewing PDF | (2021:09:65295:2:0:NEW 27 Dec 2021) 
200 Group IV, Motif 17 was only observed in Group VII, and Motif 6 was only present in Group III. 201 It is worth mentioning that the gene structure and motif organization of $C p V Q s$ were similar 202 within the same group, but diverged between groups, which also indicated that the phylogenetic 203 classification was reliable.

\section{Chromosome mapping and duplication events analysis}

205 Two $V Q$ genes ( $C p V Q 1$ and $C p V Q 2)$ could not be mapped on any chromosome, but the 206 remaining $C p V Q s$ were randomly distributed on the chromosomes, except chomosomes 15 and 20718 (Fig. 3). Chromosome 19 had the highest number of $C p V Q s(\mathrm{n}=7)$, followed by chromosome $20810(n=6)$, chromosomes $1,2,6,8$, and $14(n=3)$, chromosome $7(n=2)$, and the remaining 209 chromosomes contained one $V Q$. In addition, most $C p V Q s$ were found to be mainly located on 210 the two ends of the chromosomes.

211 To better understand the evolution mechanism of $V Q s$ in plants, the tandem duplication 212 and segmental duplication events of the CpVQ family were evaluated. Based on BLSTAp 213 results, no tandem duplication event was detected. A total of 21 pairs with $28 C p V Q s$ were 214 identified in the whole genome (Fig. 4). Moreover, groups V and IV contained six and five 215 segmental duplication events, respectively. Hence, these results suggested that segmental 216 duplications were the primary driving force responsible for the expansion of the VQ family in $C$. 217 pepo, which is consistent with previous reports.

\section{Synteny analysis of $\mathbf{V Q}$ genes}

219 To further understand the expansion mechanisms of the CpVQ family, we constructed

220

221

222

223

224

225

226

227

228

229

230

231

232

233

234

235

236

237

238

239

comparative syntenic maps of $C$. pepo associated with four representative species, including two dicots (Arabidopsis and Cucumis melo) and two monocots (O. sativa and Z. mays). Overall, the $C p V Q$ genes had the most homologous gene pairs with $C$. melo $(\mathrm{n}=27)$, followed by Arabidopsis $(\mathrm{n}=16)$. However, no homologous gene pairs were observed between C. pepo and $O$. sativa or $Z$. mays(Fig. 5). The syntenic analysis results further suggested that the $V Q$ genes are highly conserved in dicotyledonous plants.

We calculated the $\mathrm{Ka}, \mathrm{Ks}$ and $\mathrm{Ka} / \mathrm{Ks}$ ratios of all para-homologous gene pairs (Table 2) to explore the evolutionary constraints of repeated $C p V Q s$. The $\mathrm{Ka} / \mathrm{Ks}$ values of most gene pairs were less than 1.0, which indicated that these gene pairs had undergone purification selection pressure. In addition, 21 pairs of genes vaeried from 31 to 216 MYA.

\section{Expression Pattern of the CpVQ Genes in Different Tissues}

In order to explore the possible role of $C p V Q$ gene in the growth and development of $C$. pepo, we performed qRT-PCR expression analysis in three tissues of roots, stems and leaves. Expression patterns varied among the randomly selected $15 C p V Q$ genes (Fig. 6).Five $C p V Q$ genes (CaVQ1, 3,12,21 and 34) were highly expressed in the root;two $C p V Q$ genes ( $C p V Q 9$ and 22) were highly expressed in leaf and root;one $C p V Q$ genes $C p V Q 40$ were highly expressed in stem and root;three $C p V Q$ genes ( $C p V Q 16,26$ and 39) were highly expressed in stem.

\section{Expression pattern of the CpVQs under powdery mildew stress}

Several studies have shown that $V Q s$ play an important role in plant response to abiotic and biotic stress. Therefore, the expression profiles of $C p V Q s$ under powdery mildew stress were

Peer) reviewing PDF | (2021:09:65295:2:0:NEW 27 Dec 2021) 
240 evaluated next based on RNA-seq data. Most of the $C p V Q s$ were found to be downregulated at 241 early treatment timepoints, such as the members in group I and IV(Fig. 7). In addition, some 242 members were downregulated and then upregulated. A total of $15 \mathrm{CpVQs}$ from different groups 243 were randomly selected for further validation by qRT-PCR(Fig. 8). Accordingly, most of the 244 tested $C p V Q s$ were confirmed to be downregulated after powdery mildew infection, with the 245 expression of these genes being correlated with the RNA-seq data at different treatment

246

247

248

249

250

251

252

253

254

255

256

257

258

259

260

261

262

263

264

265

266

267

268

269

270

271

272

273

274

275

276

277

278

279 timepoints. Among these genes, The expression of two genes ( $C p V Q 1, C p V Q 39)$ significantly increased (more than 2-fold) at $12 \mathrm{~h}$,whereas the expression of eight genes ( $\mathrm{CpVQ3}, \mathrm{CpVQ}$, $C p V Q 12, C p V Q 16, C p V Q 21, C p V Q 22, C p V Q 36$, and $C p V Q 40)$ was significantly downregulated.In addition, three genes ( $C p V Q 26, C p V Q 33$, and $C p V Q 34)$ had a high expression value at $24 \mathrm{~h}$ after powdery mildew stress.

\section{CpVQs gene expression following abiotic stress by qRT-PCR}

To further investigate the role of $C p V Q s$ in abiotic stress responses, We randomly selected 15 $C p V Q$ genes from eight groups, and made sure their responses to the drought-,cold-, salt-, and waterlogging-stress.

Under drought treatment(Fig. 9), all $C p V Q s$ were upregulated at different treatment timepoints. The expression of six genes ( $C p V Q 1, C p V Q 14, C p V Q 26, C p V Q 33, C p V Q 34$, and $C p V Q 40$ ) significantly increased (more than 5-fold) at $12 \mathrm{~h}$, whereas $C p V Q 21$ was significantly downregulated at $12 \mathrm{~h}$ but it then increased at $24 \mathrm{~h}$. In addition, five genes ( $C p V Q 3, C p V Q 8$, $C p V Q 9, C p V Q 16$, and $C p V Q 39$ ) had a high expression value at $24 \mathrm{~h}$ after drought stress.

During cold stress(Fig. 10), the expression of eight $C p V Q s(C p V Q 1, C p V Q 16, C p V Q 22$, $C p V Q 26, C p V Q 33, C p V Q 34, C p V Q 39$, and $C p V Q 40$ ) were significantly upregulated (more than 2-fold) at $24 \mathrm{~h}$. In contrast, four genes ( $C p V Q 3, C p V Q 9, C p V Q 21$ and $C p V Q 36)$ were downregulated at $12 \mathrm{~h}$ and their levels were further decreased at $24 \mathrm{~h}$. Three members $(C p V Q 8$, $C p V Q 14$, and $C p V Q 34$ ) were downregulated at early time points but their expression were significantly increased and peaked at $24 \mathrm{~h}$.

Upon salt stress(Fig. 11), six genes (CpVQ16, CpVQ22, CpVQ26, CpVQ33, CpVQ34, and $C p V Q 40$ ) shared a similar expression trend, rapidly rising at early time points but with subsequently decreased expression. In contrast, $C p V Q 8$ and $C p V Q 12$ showed a trend of downregulation from 0 to $12 \mathrm{~h}$, but then their levels were rapidly increased. In addition, the expression of $C p V Q 21$ showed a trend of downregulation from 0 to $24 \mathrm{~h}$.

For the case of waterlogging treatment(Fig. 12), The four genes( $C p V Q 3, C p V Q 9, C p V Q 21$ and $C p V Q 26)$ upregulated in the first $12 \mathrm{~h}$ and then downregulated in the next $24 \mathrm{~h}$. In contrast, $C p V Q 8, C p V Q 12$ and $C p V Q 14$ downregulated in the first $12 \mathrm{~h}$ and then upregulated in the next 24h. Interesting, the expression of seven $C p V Q s$ (CpVQ1, CpVQ16, CpVQ22, CpVQ33, $C p V Q 34, C p V Q 39$, and $C p V Q 40$ ) were upregulated from 0 to $24 \mathrm{~h}$.

\section{Discussion}

VQ has been proven to be the main transcriptional regulator in plants. Currently, the VQ family genes have been systematically analyzed in Arabidopsis(Cheng at al., 2012), rice( Kim at al., 2013a), maize(Song et al. ,2016), tobacco(Liu et al.,2020) and cotton(Cheng et al.,2020), and 
280

281

282

283

284

285

286

287

288

289

290

291

292

293

294

295

296

297

298

299

300

301

302

303

304

305

306

307

308

309

310

311

312

313

314

315

316

317

318

responding to biotic and abiotic stresses(Jing et al.,2018;Perruc at al., 2004). However, current information on VQ characteristics in C. pepo is limited. Therefore, a comprehensive analysis of $V Q$ genes in C. pepo and their expression patterns under various non-biological and powdery mildew treatments may pave the way for a better understanding of the mechanism of plant growth and development. This will also help to select candidate genes and lay the foundation for further in-depth study of the role of different regulatory networks in plant development and stress-related processes.

\section{Conservation of the $V Q$ gene family of C. pepo}

In higher eukaryotes, genes without introns are very common(Louhichi., 2011;Liu at al.,2021). Herein, according to the gene structure, most $V Q$ genes in $C$. pepo were also found to have no introns. Only $11 \mathrm{VQs}$ had introns, among which $C p V Q 14$ contained four introns. This result is consistent with the lack of introns in $88.2 \%$ of Arabidopsis, $90 \%$ of Chinese Cabbage, and $92.3 \%$ of tomato(Cheng at al., 2012; Zhang at al., 2015; Ding at al., 2019). Subsequently, a

phylogenetic tree was constructed based on the VQ proteins of C. pepo and A. thaliana. Through the analysis of the phylogenetic and structural features of the VQ domains, and these proteins were divided into 8 clades(I-VIII) based on the nomenclature of the A. thaliana VQs. This analysis revealed that $V Q s$ with introns are located in different subfamilies, suggesting that these introns appear relatively independently. Comparative these plants (Cicer arietinum and Medicago truncatula, A. thaliana and lower plants, moss) indicate that most $V Q$ genes have lost introns during the long evolutionary period. Taken together, $V Q s$ identified in C. pepo and in other species provide a certain reference value for the evolution of introns in plants. The average length of the $C p V Q$ is 219.3 amino acids, a majority of the C. pepo VQ genes are intronless so they encode relatively small proteins with fewer than 300 amino acid residues, which is highly similar to those reported for Nicotiana tabacum, Arabidopsis, tomato, O. sativa, and other plants(Liu at al., 2020; Cheng at al., 2012; Ding at al., 2019; Kim at al., 2013a). Noteworthy, $C p V Q 4, C p V Q 14, C p V Q 23$, and $C p V Q 32$ were all found to be close to 400 amino acids.

The genetic structure of the $V Q$ gene and the conserved Motif not only appear in higher plants such as Arabidopsis, O. sativa, Z. mays, among others, but also in lower plants such as moss, which also implies their ancient origins in the evolutionary history and their important role in plant development(Jing at al., 2015; Song at al., 2016). Moreover, all 44 VQ proteins of C.pepo were found to harbor conserved VQ domains and contained the same type of Motifs, implying that in the same branch of the evolutionary tree, the closer the genes of VQ protein are, the more similar the gene structure is. Motif 1 was identified as the core Motif that composes the VQ domain, which was included in all pumpkin VQ proteins, thereby endowing the pumpkin VQ protein with specific biological functions. These results were in agreement with those reported for tomato and soybean species(Ding at al., 2019; Wang at al., 2019). In addition, VQ proteins with similar Motif composition were also found to be located in the same sub-branch of the evolutionary tree, as the Motifs composition between different branches was different.

\section{Expansion mechanism of the C. pepo VQ family}


319 Genome replication events play an important role in expanding the size of the genome and 320 diversifying gene functions, as replication events can result in genes with new

321 functions(Rensing., 2014). In higher plants, tandem repeat events and chromosome fragment

322 replication events are the main processes contributing to gene family expansion(Wang at al.,

323 2012).Previous studies have shown that diverse WGD events lead to the different sizes of plant

324 genomes(Adams et al.,2005).In this study, $44 V Q$ genes were identified in C. pepo, which is a

325 slightly high number of genes as compared with other species, such as Arabidopsis with $34 \mathrm{VQ}$

326 members, or $O$. sativa and Vitis vinifera with 39 and $18 V Q s$, respectively(Cheng at al.,2012;Kim

327 at al.,2013a; Wang et al.,2015a). Nevertheless, the number of VQ members in C. pepo was lower

328 than initially predicted while considering its genome size of $261 \mathrm{Mb}$, as compared with the 125 ,

$329389,486 \mathrm{Mb}$ of Arabidopsis, O. sativa, and V. vinifera, respectively. Therefore, it can be

330 concluded that there is no necessary connection between the size of the genome and the number

331 of family members. Previous studies have shown that chromosome fragment replication is

332 considered to be the main mechanism of $V Q$ gene expansion(Tang at al., 2008); thus, the

333 evolutionary process can explain the number of specific $V Q$ genes in a species, not the size of the

334

335 genome. The main process for the expansion of the C. pepo VQ family was found to be chromosome fragment duplication events, which is consistent with the results of previous

336 studies. Among the $44 \mathrm{CpVQs}$, a total of 28 members participated in 21 chromosome fragment

337 duplication events, accounting for $63.6 \%$, and no tandem duplication event was identified. This

338 is consistent with the expansion of the VQ family in Brassica napus, which is driven by chromosome fragment duplication, with tandem repeat events as the second driving force(Zou at al., 2021). Gene duplication can produce gene function redundancy, and these repeated genes can develop different gene expression patterns. We calculated the $\mathrm{Ka}$, $\mathrm{Ks}$ and $\mathrm{Ka} / \mathrm{Ks}$ ratios of all para-homologous gene pairs to explore the evolutionary constraints of repeated CpVQs. The $\mathrm{Ka} / \mathrm{Ks}$ values of most gene pairs were less than 1.0 , which indicated that these gene pairs had undergone purification selection pressure.In the present study, some synlinear genes showed different expression patterns, such as $C p V Q 3$ and $C p V Q 33, C p V Q 9$ and $C p V Q 21$, including under drought treatment, suggesting that these homologous genes in C. pepo may have different functions in regulation the normal growth and development of plants.

Previous studies have shown that members of the VQ family play an important role in the entire plant development process and respond to various biotic and abiotic stresses. Molecular genetic evidence suggests that the plant VQ protein may be an important regulator of disease resistance and tolerance(Wang at al., 2015). In this study, we analyzed the expression level of $C p V Q$ gene in different tissues of C. pepo (Fig.6). The results showed that most genes were differentially expressed in the tissues we analyzed. It indicates that the $C p V Q$ gene may play an important role in the growth and development of these organs or tissues. In addition, based on transcriptome data, the expression $V Q s$ in $C$. pepo leaves under powdery mildew treatments at different timepoints was evaluated(as observed in Fig. 7). Most VQ members showed a significant decline in expression after $12 \mathrm{~h}$ of powdery mildew treatment, however, the expression patterns of the 
359

360

361

362

363

364

365

366

367

368

369

370

371

372

373

374

375

376

377

378

379

380

381

382

383

384

385

386

387

388

389

390

391

392

393

394

395

396

397

398

third group is different from the other groups. In the third group, except $C p V Q 20$, all were significantly up-regulated upon powdery mildew infection, and 3 genes (CpVQ18, 27 and 38) had higher expression at $24 \mathrm{~h}$, which may be because these genes responded late to powdery mildew stress. Studies have shown that SIB1(AtVQ23) and SIB2(AtVQ16) function as activators in plant defense against necrotrophic pathogens(Lai et al.,2011), so the $C p V Q$ genes classified in the third group may have the same function. In contrast, after 24 hours of powdery mildew treatment, the expression of most members in other groups decreased significantly. Similar results have been reported in grapevine that $V v V Q$ genes are quickly responsive to powdery mildew stresses(Wang et al.,2015). Therefore, the members of the VQ family in C. pepo may play a key role in the powdery mildew signaling pathway. Moreover, analysis of $V Q s$ expression under different abiotic stresses further demonstrated that the members of the VQ family are also significantly induced under different stresses. In this study, four $C p V Q$ genes $(C p V Q 3,9,16$, and 22 ) significantly upregulated during drought stress(as observed in Fig. 9), which results are similar to the $O s V Q$ genes that twenty-two $O s V Q$ genes are upregulated under drought stress(Kim et al.,2013a). Under cold stress(as observed in Fig. 10), five CaVQ genes (CaVQ1, 22, 33, 39 and 40) were significantly induced. Similar results have been reported in Chinese cabbage that $B r V Q$ genes are quickly responsive to cold stresses(Zhang et al.,2015). In addition, the $V Q$ genes are also sensitive to salt changes. Two $C p V Q$ genes ( $C a V Q 3$ and 9) were upregulated during salt treatment(as observed in Fig. 11). Similar changes occurred in Arabidopsis. AtVQ9 and AtVQ15 were significantly expressed under salt stress. Seven CaVQ genes (CaVQ1, 16, 22, 33, 34, 39 and 40) were upregulated at waterlogging treatment(as observed in Fig. 12).In summary, CpVQ members may be involved in regulating the response of plants to various abiotic stresses and powdery mildew stress, and their response mechanisms may be complex and diverse.

In summary, this study has systematically analyzed the evolutionary relationship, conserved structure, and expression patterns of the members of the VQ family of C. pepo at the whole genome level. The selection of candidate genes can provide reference for future investigations.

\section{Conclusions}

In conclusion, this study provides the first comprehensive and systematic analysis of the $44 V Q$ genes identified in C. pepo genome. All $C p V Q$ genes can be divided into eight groups (I-VIII), and were found to expand by chromosome fragment duplication events. RNA-seq analysis further showed that half of the $V Q s$ have significantly different expression patterns at different timepoints of powdery mildew infection. Therefore, the members of the VQ family in C. pepo may play a key role in the powdery mildew signaling pathway. Genetic analysis data further confirmed that the VQ family members respond to different abiotic stresses. Taken together, these findings provide a theoretical basis for further research on the functions of $C p V Q s$.

\section{References}


399

400

401

402

403

404

405

406

407

408

409

410

411

412

413

414

415

416

417

418

419

420

421

422

423

424

425

426

427

428

429

430

431

432

433

434

435

436

437

Adams et al.(2005)Adams KL, Wendel JF. Polyploidy and genome evolution in plants. Curr Opin Plant Biol. 2005 Apr;8(2):135-41. doi: 10.1016/j.pbi.2005.01.001. PMID: 15752992. Bailey et al.(2015)Bailey TL, Johnson J, Grant CE, Noble WS. The MEME Suite. Nucleic Acids Res. 2015 Jul 1;43(W1):W39-49. doi: 10.1093/nar/gkv416. Epub 2015 May 7. PMID: 25953851; PMCID: PMC4489269.

Chen et al. (2020) Chen C, Chen H, Zhang Y, Thomas HR, Frank MH, He Y, Xia R. TBtools: An Integrative Toolkit Developed for Interactive Analyses of Big Biological Data. Mol Plant. 2020 Aug 3;13(8):1194-1202. doi: 10.1016/j.molp.2020.06.009. Epub 2020 Jun 23. PMID: 32585190 .

Chen et al.(2018)Chen J, Wang H, Li Y, Pan J, Hu Y, Yu D. Arabidopsis VQ10 interacts with WRKY8 to modulate basal defense against Botrytis cinerea. J Integr Plant Biol. 2018 Oct;60(10):956-969. doi: 10.1111/jipb.12664. Epub 2018 Jul 10. PMID: 29727045. Cheng et al. (2012) Cheng Y, Zhou Y, Yang Y, Chi YJ, Zhou J, Chen JY, Wang F, Fan B, Shi $\mathrm{K}$, Zhou YH, Yu JQ, Chen Z. Structural and functional analysis of VQ motif-containing proteins in Arabidopsis as interacting proteins of WRKY transcription factors. Plant Physiol. 2012 Jun;159(2):810-25. doi: 10.1104/pp.112.196816. Epub 2012 Apr 24. PMID: 22535423; PMCID: PMC3375943.

Cheng et al. (2020) Chen P, Wei F, Cheng S, Ma L, Wang H, Zhang M, Mao G, Lu J, Hao P, Ahmad A, Gu L, Ma Q, Wu A, Wei H, Yu S. A comprehensive analysis of cotton VQ gene superfamily reveals their potential and extensive roles in regulating cotton abiotic stress. BMC Genomics. 2020 Nov 16;21(1):795. doi: 10.1186/s12864-020-07171-z. PMID: 33198654; PMCID: PMC7667805.

Chi et al. (2013) Chi Y, Yang Y, Zhou Y, Zhou J, Fan B, Yu JQ, Chen Z. Protein-protein interactions in the regulation of WRKY transcription factors. Mol Plant. 2013 Mar;6(2):287-300. doi: 10.1093/mp/sst026. Epub 2013 Mar 2. PMID: 23455420.

Caoet al. (2018) Cao Y, Meng D, Abdullah M, Jin Q, Lin Y, Cai Y. Genome Wide Identification, Evolutionary, and Expression Analysis of VQ Genes from Two Pyrus Species. Genes (Basel). 2018 Apr 23;9(4):224. doi: 10.3390/genes9040224. PMID: 29690608; PMCID: PMC5924566.

Ding et al. (2019) Ding H, Yuan G, Mo S, Qian Y, Wu Y, Chen Q, Xu X, Wu X, Ge C. Genome-wide analysis of the plant-specific VQ motif-containing proteins in tomato (Solanum lycopersicum) and characterization of SIVQ6 in thermotolerance. Plant Physiol Biochem. 2019 Oct;143:29-39. doi: 10.1016/j.plaphy.2019.08.019. Epub 2019 Aug 27. PMID: 31479880. Fujita et al. (2006) Fujita M, Fujita Y, Noutoshi Y, Takahashi F, Narusaka Y, YamaguchiShinozaki K, Shinozaki K. Crosstalk between abiotic and biotic stress responses: a current view from the points of convergence in the stress signaling networks. Curr Opin Plant Biol. 2006 Aug;9(4):436-42. doi: 10.1016/j.pbi.2006.05.014. Epub 2006 Jun 8. PMID: 16759898.

Hu et al. (2013) Hu Y, Chen L, Wang H, Zhang L, Wang F, Yu D. Arabidopsis transcription factor WRKY8 functions antagonistically with its interacting partner VQ9 to modulate salinity 
438 stress tolerance. Plant J. 2013 Jun;74(5):730-45. doi: 10.1111/tpj.12159. Epub 2013 Apr 1.

439 PMID: 23451802.

440 Jing et al.(2018)Jiang SY, Sevugan M, Ramachandran S. Valine-glutamine (VQ) motif coding 441 genes are ancient and non-plant-specific with comprehensive expression regulation by various 442 biotic and abiotic stresses. BMC Genomics. 2018 May 9;19(1):342. doi: 10.1186/s12864-018443 4733-7. PMID: 29743038; PMCID: PMC5941492.

444 Jing et al.(2015)Jing Y, Lin R. The VQ Motif-Containing Protein Family of Plant-Specific 445 Transcriptional Regulators. Plant Physiol. 2015 Sep;169(1):371-8. doi: 10.1104/pp.15.00788. 446 Epub 2015 Jul 28. PMID: 26220951; PMCID: PMC4577417.

447 Kim et al.(2013a)Kim DY, Kwon SI, Choi C, Lee H, Ahn I, Park SR, Bae SC, Lee SC, Hwang 448 DJ. Expression analysis of rice VQ genes in response to biotic and abiotic stresses. Gene. 2013 449 Oct 25;529(2):208-14. doi: 10.1016/j.gene.2013.08.023. Epub 2013 Aug 16. PMID: 23958655. 450 Kim et al. (2013b) Kim MY, Yang JK, Lee T, Lee SH. Divergence of flowering-related genes 451 in three legume species. Plant Genome. 2013b;6(3):841-856. doi:

452 10.3835/plantgenome2013.03.0008.

453 Lai et al. (2011) Lai Z, Li Y, Wang F, Cheng Y, Fan B, Yu JQ, Chen Z. Arabidopsis sigma

454

455

456

457

458

459

460

461

462

463

464

465

466

467

468

469

470

471

472

473

474 factor binding proteins are activators of the WRKY33 transcription factor in plant defense. Plant Cell. 2011 Oct;23(10):3824-41. doi: 10.1105/tpc.111.090571. Epub 2011 Oct 11. PMID: 21990940 ; PMCID: PMC3229152.

Lei et al. (2017) Lei R, Li X, Ma Z, Lv Y, Hu Y, Yu D. Arabidopsis WRKY2 and WRKY34 transcription factors interact with VQ20 protein to modulate pollen development and function. Plant J. 2017 Sep;91(6):962-976. doi: 10.1111/tpj.13619. Epub 2017 Jul 20. PMID: 28635025. Lei et al. (2018) Lei R, Ma Z, Yu D. WRKY2/34-VQ20 Modules in Arabidopsis thaliana Negatively Regulate Expression of a Trio of Related MYB Transcription Factors During Pollen Development. Front Plant Sci. 2018 Mar 19;9:331. doi: 10.3389/fpls.2018.00331. PMID: 29616054; PMCID: PMC5867338.

Livak et al. (2001) Livak KJ, Schmittgen TD. Analysis of relative gene expression data using real-time quantitative PCR and the 2(-Delta Delta C(T)) Method. Methods. 2001 Dec;25(4):4028. doi: 10.1006/meth.2001.1262. PMID: 11846609.

Liu et al. (2020) Liu C, Liu H, Zhou C, Timko MP. Genome-Wide Identification of the VQ Protein Gene Family of Tobacco (Nicotiana tabacum L.) and Analysis of Its Expression in Response to Phytohormones and Abiotic and Biotic Stresses. Genes (Basel). 2020 Mar 7;11(3):284. doi: 10.3390/genes1 1030284. PMID: 32156048; PMCID: PMC7140788. Liu et al. (2021) Liu H, Lyu HM, Zhu K, Van de Peer Y, Max Cheng ZM. The emergence and evolution of intron-poor and intronless genes in intron-rich plant gene families. Plant J. 2021 Feb;105(4):1072-1082. doi: 10.1111/tpj.15088. Epub 2021 Feb 9. PMID: 33217085; PMCID: PMC7116809. 
475 Louhichi et al. (2011) Louhichi A, Fourati A, Rebaï A. IGD: a resource for intronless genes in 476 the human genome. Gene. 2011 Nov 15;488(1-2):35-40. doi: 10.1016/j.gene.2011.08.013. Epub 4772011 Sep 2. PMID: 21914464.

478 Luo et al. (2005) Luo M, Dennis ES, Berger F, Peacock WJ, Chaudhury A. MINISEED3 479 (MINI3), a WRKY family gene, and HAIKU2 (IKU2), a leucine-rich repeat (LRR) KINASE 480 gene, are regulators of seed size in Arabidopsis. Proc Natl Acad Sci U S A. 2005 Nov 481 29;102(48):17531-6. doi: 10.1073/pnas.0508418102. Epub 2005 Nov 17. PMID: 16293693; 482 PMCID: PMC1297679.

483 Obrero et al.(2011)Obrero A, Die JV, Román B, Gómez P, Nadal S, González-Verdejo CI. 484 Selection of reference genes for gene expression studies in zucchini (Cucurbita pepo) using 485 qPCR. J Agric Food Chem. 2011 May 25;59(10):5402-11. doi: 10.1021/jf200689r. Epub 2011 486 Apr 22. PMID: 21476515.

487 Perruc et al.(2004)Perruc E, Charpenteau M, Ramirez BC, Jauneau A, Galaud JP, Ranjeva R, 488 Ranty B. A novel calmodulin-binding protein functions as a negative regulator of osmotic stress 489 tolerance in Arabidopsis thaliana seedlings. Plant J. 2004 May;38(3):410-20. doi:

490 10.1111/j.1365-313X.2004.02062.x. PMID: 15086802.

491 Punta et al. (2004) Punta M, Coggill PC, Eberhardt RY, Mistry J, Tate J, Boursnell C, Pang N, 492 Forslund K, Ceric G, Clements J. The Pfam protein families database. Nucleic Acids Research. 493 2004;28(1):263-266. doi: 10.1093/nar/gkh121.

494 Rensing et al. (2014) Rensing SA. Gene duplication as a driver of plant morphogenetic 495 evolution. Curr Opin Plant Biol. 2014 Feb;17:43-8. doi: 10.1016/j.pbi.2013.11.002. Epub 2013 496 Nov 28. PMID: 24507493.

497 Song et al.(2016)Song W, Zhao H, Zhang X, Lei L, Lai J. Genome-Wide Identification of VQ 498 Motif-Containing Proteins and their Expression Profiles Under Abiotic Stresses in Maize. Front 499 Plant Sci. 2016 Jan 5;6:1177. doi: 10.3389/fpls.2015.01177. PMID: 26779214; PMCID: 500 PMC4700186.

501 Tamura et al. (2011) Tamura K, Peterson D, Peterson N, Stecher G, Nei M, Kumar S. 502 MEGA5: molecular evolutionary genetics analysis using maximum likelihood, evolutionary 503 distance, and maximum parsimony methods. Mol Biol Evol. 2011 Oct;28(10):2731-9. doi:

504 10.1093/molbev/msr121. Epub 2011 May 4. PMID: 21546353; PMCID: PMC3203626.

505 Tang et al. (2008) Tang H, Bowers JE, Wang X, Ming R, Alam M, Paterson AH. Synteny and 506 collinearity in plant genomes. Science. 2008 Apr 25;320(5875):486-8. doi:

507 10.1126/science.1153917. PMID: 18436778.

508 Wang et al. (2017) Wang J, Sun P, Li Y, Liu Y, Yu J, Ma X, Sun S, Yang N, Xia R, Lei T, Liu 509 X, Jiao B, Xing Y, Ge W, Wang L, Wang Z, Song X, Yuan M, Guo D, Zhang L, Zhang J, Jin D, 510 Chen W, Pan Y, Liu T, Jin L, Sun J, Yu J, Cheng R, Duan X, Shen S, Qin J, Zhang MC, 511 Paterson AH, Wang X. Hierarchically Aligning 10 Legume Genomes Establishes a Family-Level 512 Genomics Platform. Plant Physiol. 2017 May;174(1):284-300. doi: 10.1104/pp.16.01981. Epub 5132017 Mar 21. PMID: 28325848; PMCID: PMC5411148. 
514 Wang et al. (2010a) Wang D, Zhang Y, Zhang Z, Zhu J, Yu J. KaKs_Calculator 2.0: a toolkit 515 incorporating gamma-series methods and sliding window strategies. Genomics Proteomics 516 Bioinformatics. 2010 Mar;8(1):77-80. doi: 10.1016/S1672-0229(10)60008-3. PMID: 20451164; 517 PMCID: PMC5054116.

518 Wang et al. (2010) Wang A, Garcia D, Zhang H, Feng K, Chaudhury A, Berger F, Peacock 519 WJ, Dennis ES, Luo M. The VQ motif protein IKU1 regulates endosperm growth and seed size 520 in Arabidopsis. Plant J. 2010 Aug;63(4):670-9. doi: 10.1111/j.1365-313X.2010.04271.x. PMID: 52120545893.

522 Wang et al. (2015) Wang H, Hu Y, Pan J, Yu D. Arabidopsis VQ motif-containing proteins 523 VQ12 and VQ29 negatively modulate basal defense against Botrytis cinerea. Sci Rep. 2015 Sep 524 23;5:14185. doi: 10.1038/srep14185. PMID: 26394921; PMCID: PMC4585807.

525 Wang et al. (2015a) Wang M, Vannozzi A, Wang G, Zhong Y, Corso M, Cavallini E, Cheng 526 ZM. A comprehensive survey of the grapevine VQ gene family and its transcriptional correlation 527 with WRKY proteins. Front Plant Sci. 2015 Jun 12;6:417. doi: 10.3389/fpls.2015.00417. PMID: 528 26124765; PMCID: PMC4464145.

529 Wang et al. (2019) Wang Y, Jiang Z, Li Z, Zhao Y, Tan W, Liu Z, Cui S, Yu X, Ma J, Wang

$530 \mathrm{G}, \mathrm{Li}$ W. Genome-wide identification and expression analysis of the VQ gene family in soybean 531 (Glycine max). PeerJ. 2019 Aug 21;7:e7509. doi: 10.7717/peerj.7509. PMID: 31497394; 532 PMCID: PMC6708371.

533 Wang et al. (2012a) Wang Y, Tang H, Debarry JD, Tan X, Li J, Wang X, Lee TH, Jin H, 534 Marler B, Guo H, Kissinger JC, Paterson AH. MCScanX: a toolkit for detection and evolutionary 535 analysis of gene synteny and collinearity. Nucleic Acids Res. 2012 Apr;40(7):e49. doi:

536 10.1093/nar/gkr1293. Epub 2012 Jan 4. PMID: 22217600; PMCID: PMC3326336.

537 Wang et al. (2012) Wang Y, Wang X, Paterson AH. Genome and gene duplications and gene 538 expression divergence: a view from plants. Ann N Y Acad Sci. 2012 May;1256:1-14. doi:

539 10.1111/j.1749-6632.2011.06384.x. Epub 2012 Jan 18. PMID: 22257007.

540 Wilkins et al. (1999) Wilkins MR, Gasteiger E, Bairoch A, Sanchez JC, Williams KL, Appel 541 RD, Hochstrasser DF. Protein identification and analysis tools in the ExPASy server. Methods 542 Mol Biol. 1999;112:531-52. doi: 10.1385/1-59259-584-7:531. PMID: 10027275.

543 Wray et al. (2003) Wray GA, Hahn MW, Abouheif E, Balhoff JP, Pizer M, Rockman MV,

544 Romano LA. The evolution of transcriptional regulation in eukaryotes. Mol Biol Evol. 2003 545 Sep;20(9):1377-419. doi: 10.1093/molbev/msg140. Epub 2003 May 30. PMID: 12777501. 546 Ye et al. (2016) Ye YJ, Xiao YY, Han YC, Shan W, Fan ZQ, Xu QG, Kuang JF, Lu WJ, 547 Lakshmanan P, Chen JY. Banana fruit VQ motif-containing protein5 represses cold-responsive 548 transcription factor MaWRKY26 involved in the regulation of JA biosynthetic genes. Sci Rep. 549 2016 Mar 23;6:23632. doi: 10.1038/srep23632. PMID: 27004441; PMCID: PMC4804309. 550 Zhang et al. (2015) Zhang G, Wang F, Li J, Ding Q, Zhang Y, Li H, Zhang J, Gao J. Genome551 Wide Identification and Analysis of the VQ Motif-Containing Protein Family in Chinese 
552 Cabbage (Brassica rapa L. ssp. Pekinensis). Int J Mol Sci. 2015 Dec 2;16(12):28683-704. doi:

553 10.3390/ijms161226127. PMID: 26633387; PMCID: PMC4691074.

554 Zou et al. (2021) Zou Z, Liu F, Huang S, Fernando WGD. Genome-Wide Identification and

555 Analysis of the Valine-Glutamine Motif-Containing Gene Family in Brassica napus and

556 Functional Characterization of BnMKS1 in Response to Leptosphaeria maculans.

557 Phytopathology. 2021 Feb;111(2):281-292. doi: 10.1094/PHYTO-04-20-0134-R. Epub 2021 Feb

558 8. PMID: 32804045.

559

560 
Figure 1

Phylogenetic tree analysis of the VQ genes in C.pepo and Arabidopsis thaliana.

The clusters were designated as group I-VIII and indicated in a specific color.

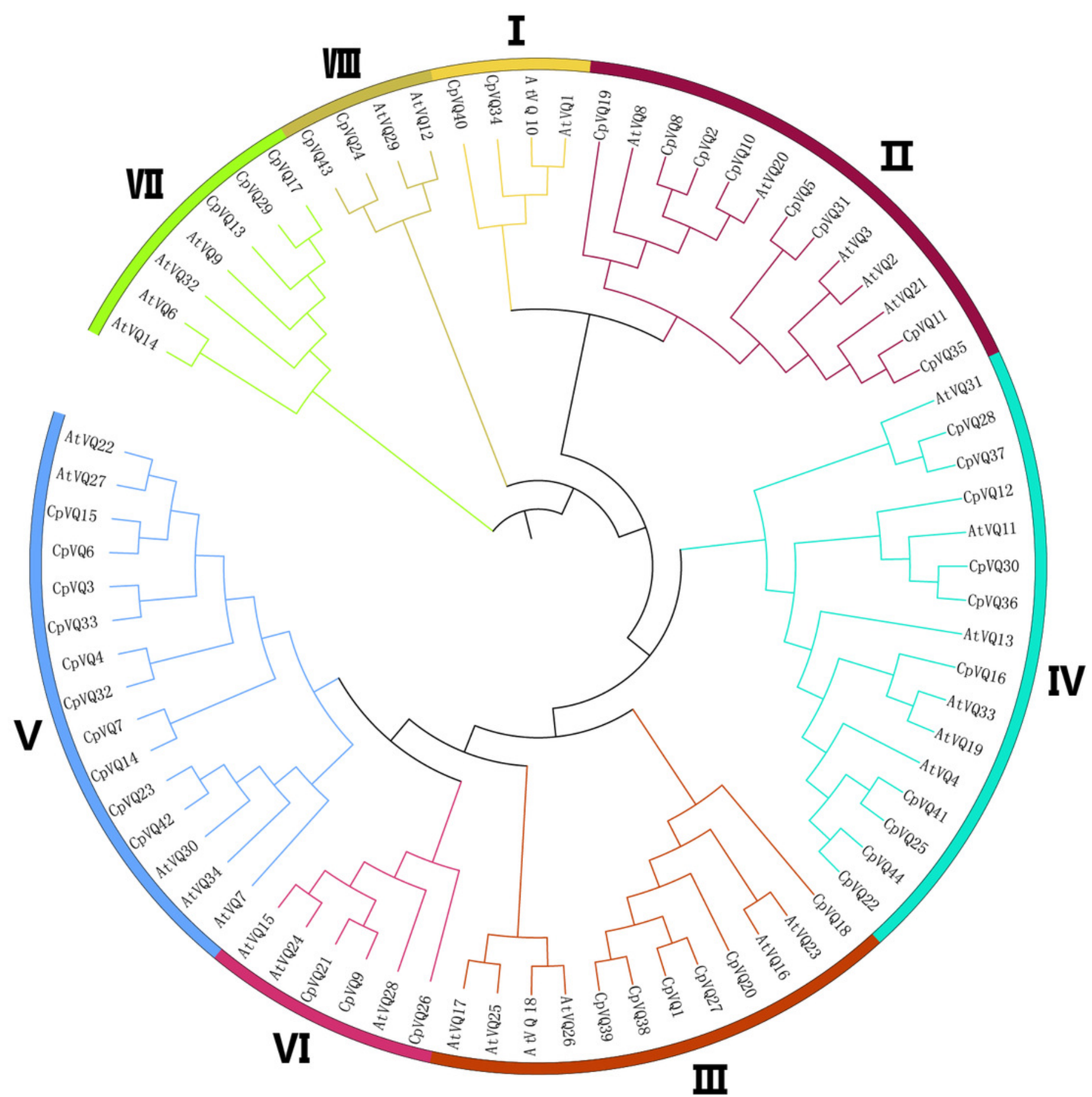


Figure 2

Phylogenetic tree, conserved motifs and gene structure in CpVQs.

(A) Phylogenetic relationships (B) Conserved motifs of the CpVQs. Each motif is represented by a number in colored box. (C) Exon/intron structures of CpVQ genes.
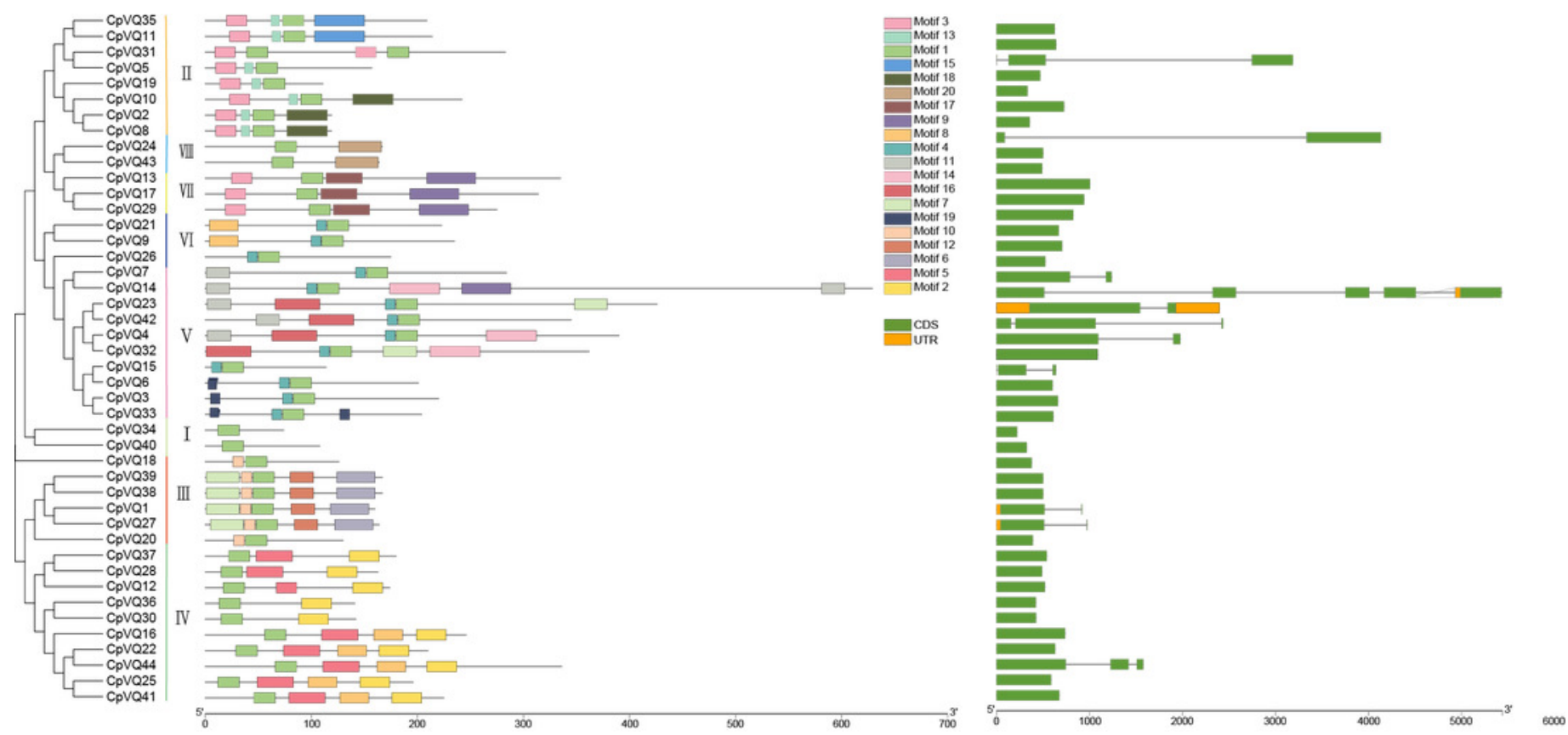
Figure 3

Chromosome location in C.pepo.

Chromosome numbers were indicated above each chromosome. The size of a chromosome was indicated by its relative length. 

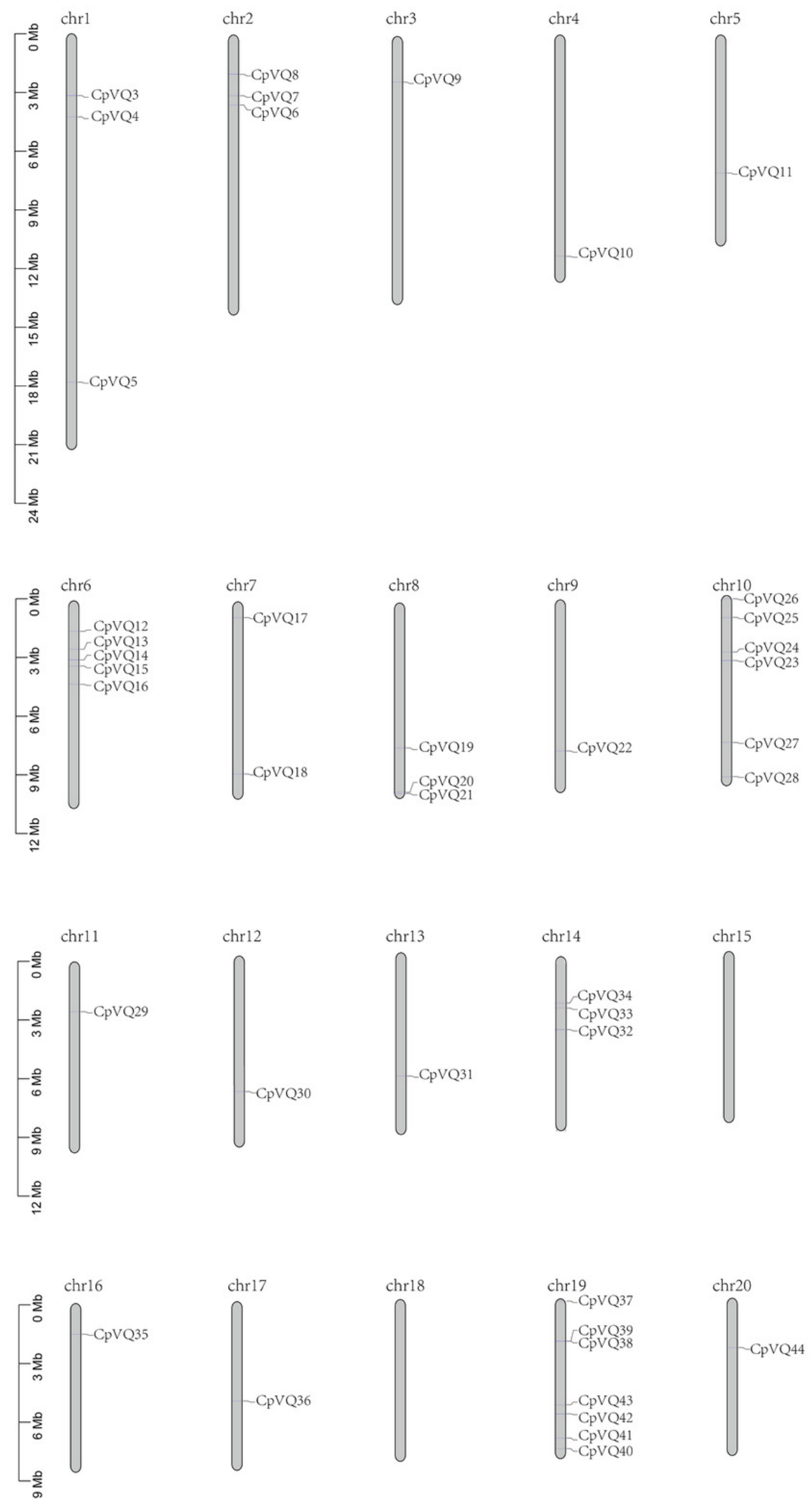
Figure 4

Syntenic analysis of $V Q$ genes

Gray lines in the background indicate the collinear blocks within the C.pepo and oneself genomes, while the dark grey lines highlight the syntenic VQ gene pairs. 


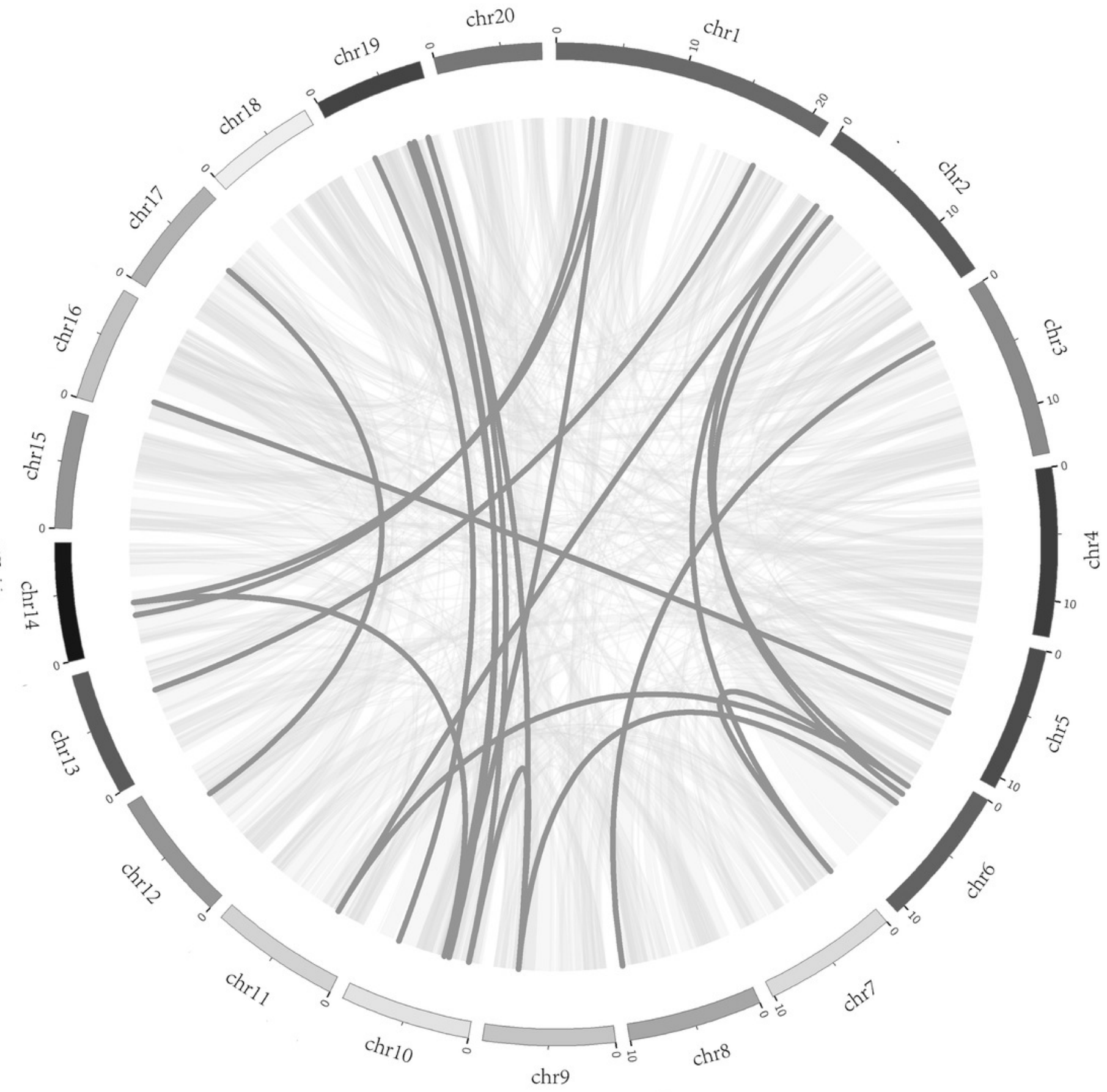




\section{Figure 5}

Synteny analysis of $V Q$ genes between C.pepo and plant species.

Synteny analysis of the VQ genes between(A)C.pepo and Arabidopsis thaliana;(B)C.pepo and Cucumis melo;(C) C.pepo and Oryza sativa;(D) C.pepo and Zea mays.Gray lines in the background indicate the collinear blocks within the C.pepo and other plant genomes, while the black lines highlight the syntenic VQ gene pairs. 

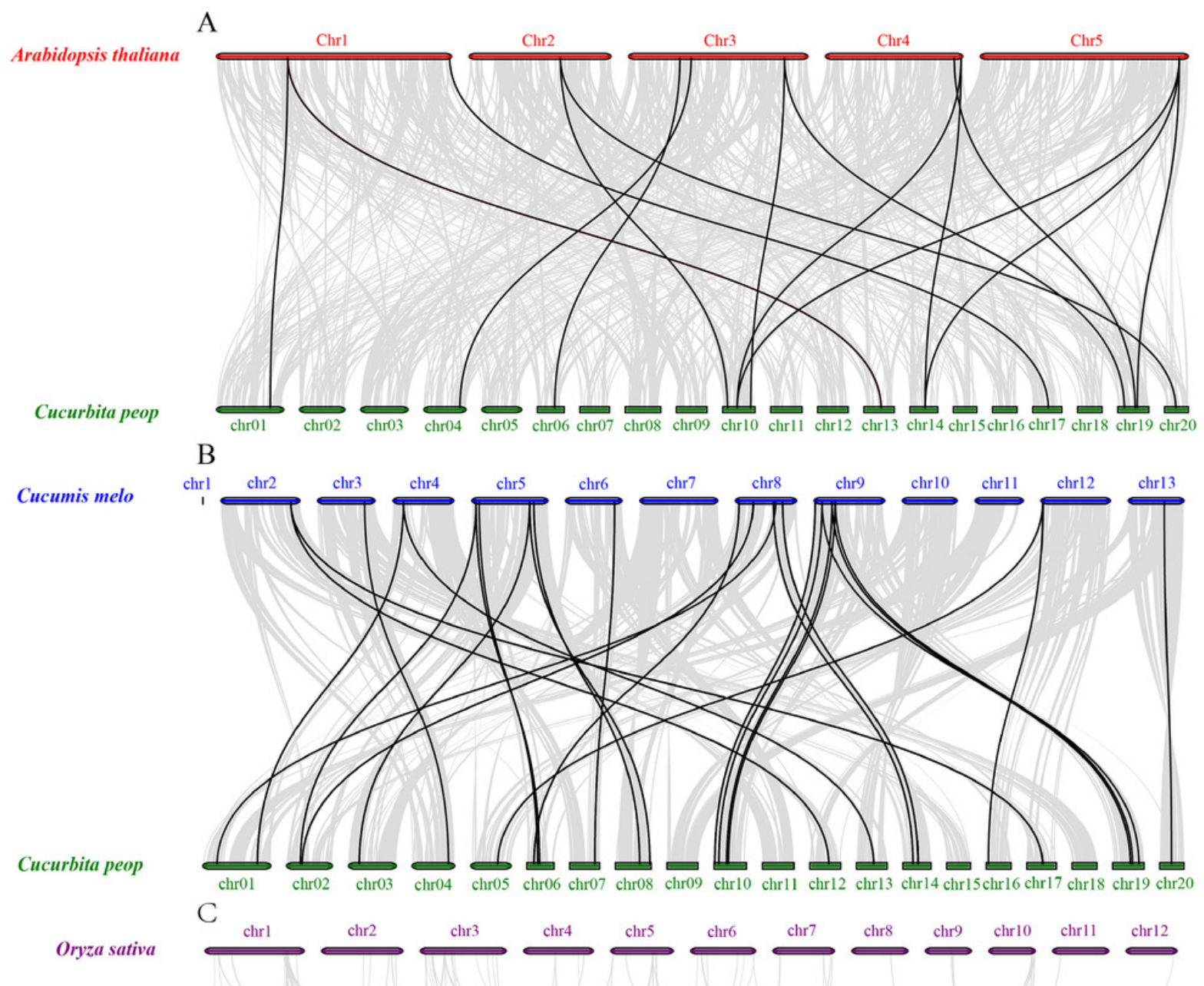

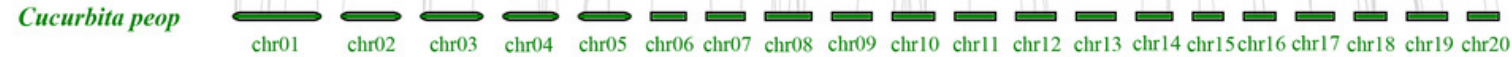

$\mathrm{D}$

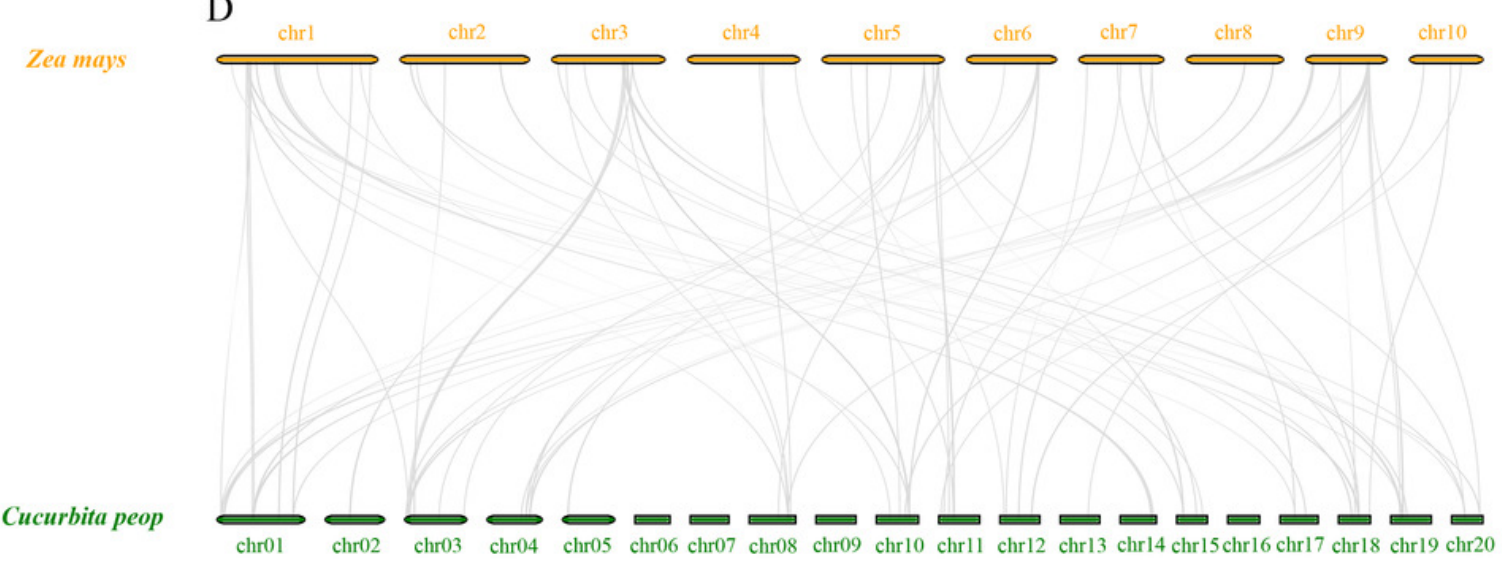


Figure 6

Expression analysis of the $C p V Q$ genes in different tissues of $C$.pepo

The surveyed tissues include root,stem, leaf. The $2^{-\Delta \Delta c t}$ method was used to calculate the expression levels of target genes in different tissues. 


\section{Different tissues}
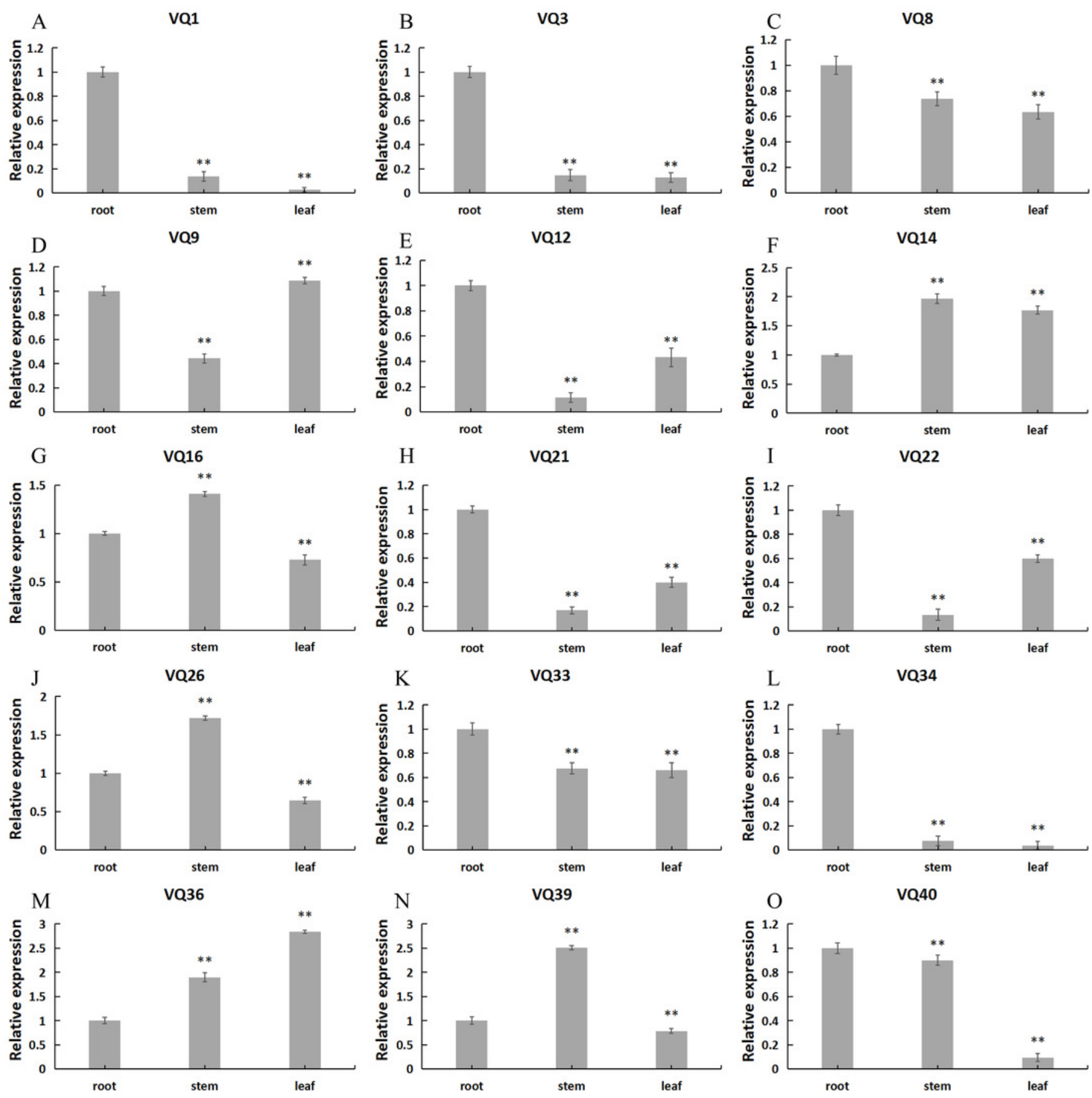


\section{Figure 7}

Expression profiles of $C p V Q s$ under powdery mildew stress based on the RNA-seq data.

The gene expression values are square-root transformed fragments per kilo-bases per million mapped reads (FPKM). Different colors in map represent gene transcript abundance values as shown in the color bar. 


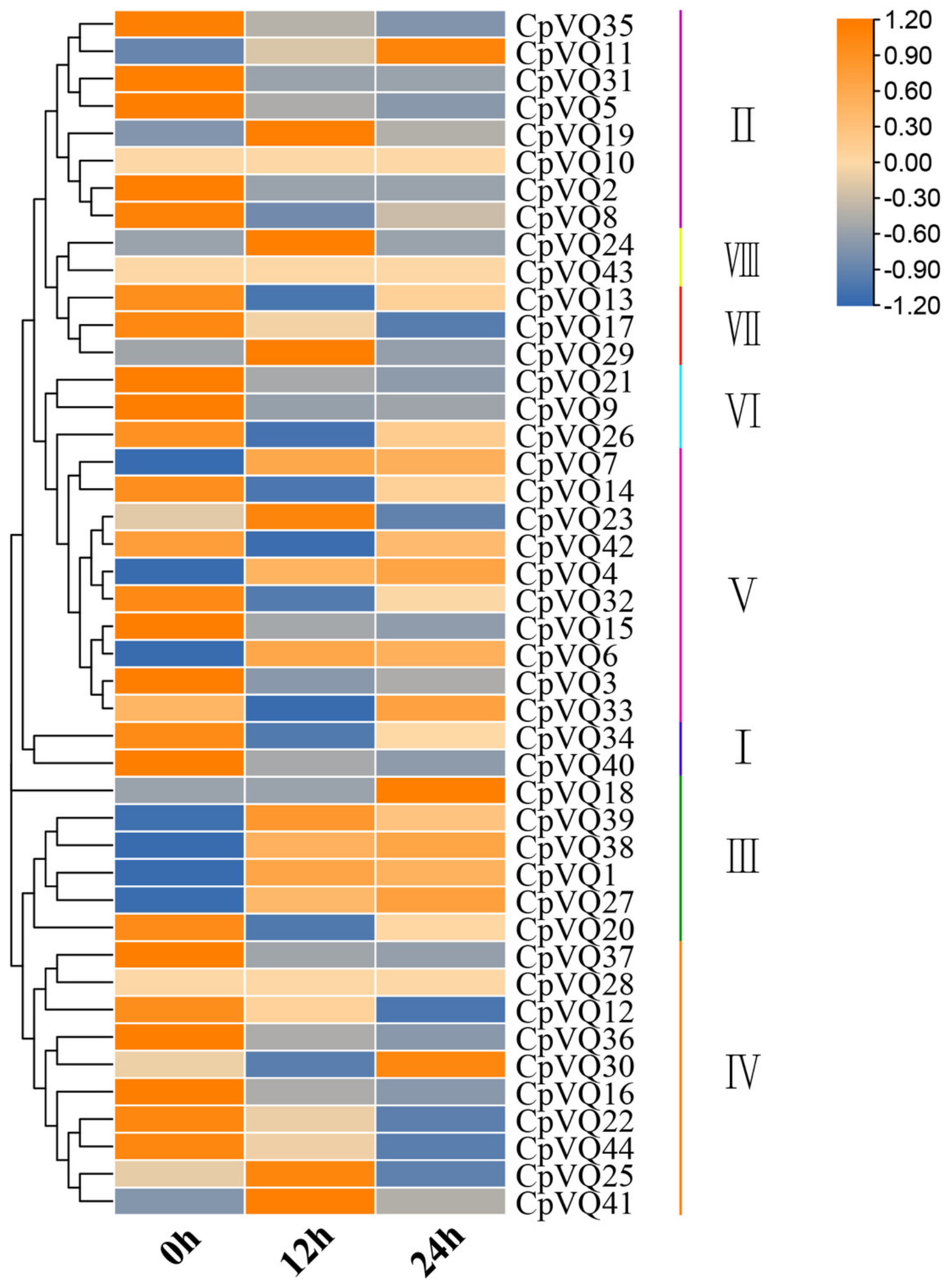


Figure 8

qRT-PCR validation of $V Q$ genes in the response to powdery mildew treatment.

Stress treatments and time course are described in "Materials \& Methods". (A-O) represent different genes that were evaluated by qRT-PCR. Asterisks indicate statistically significant differences between the stressed samples and counterpart controls $(* p<0.05, * * p<0.01)$. 


\section{powdery mildew}
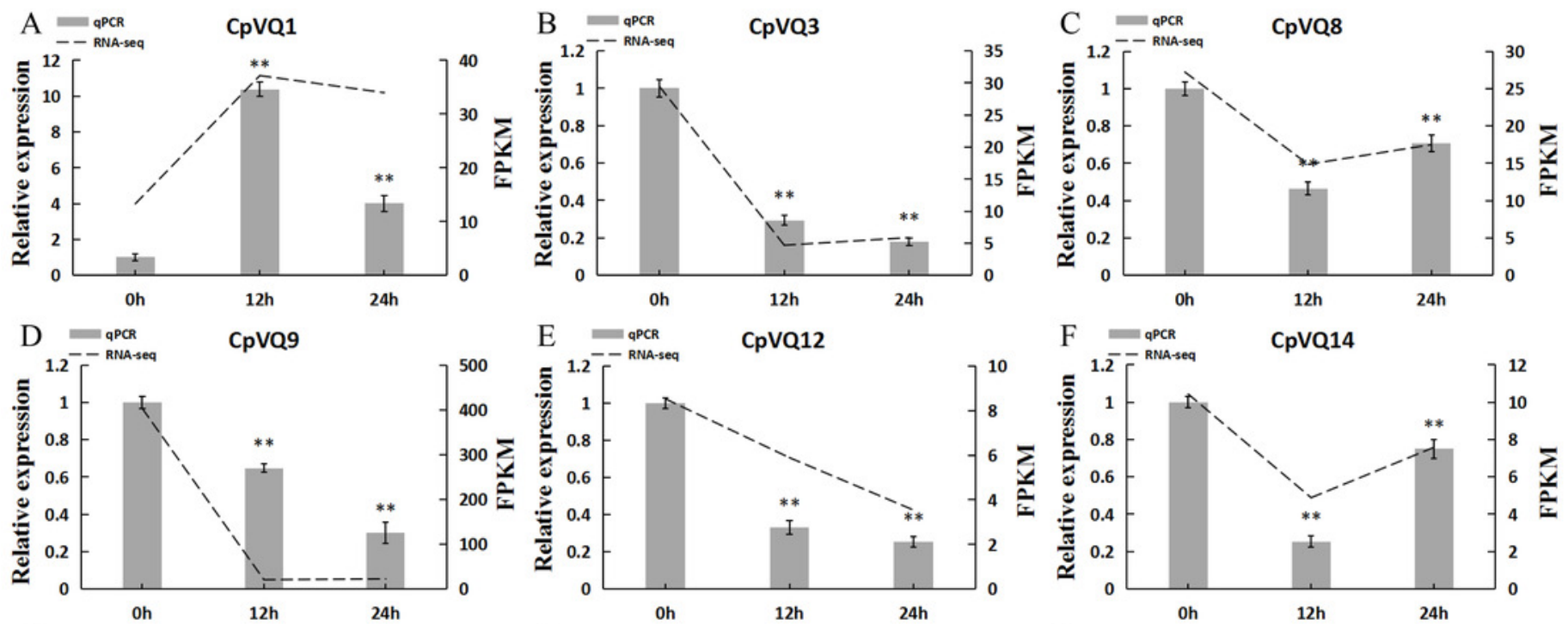

$\mathrm{E}=\mathrm{qPCR}$

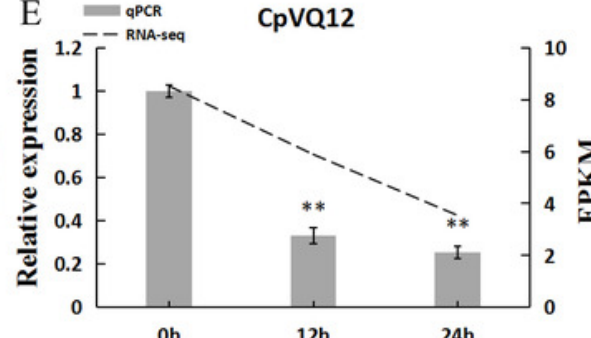

$\mathrm{F} \underset{\mathrm{qPCR}}{\mathrm{Q}} \mathrm{CpVQ14}$
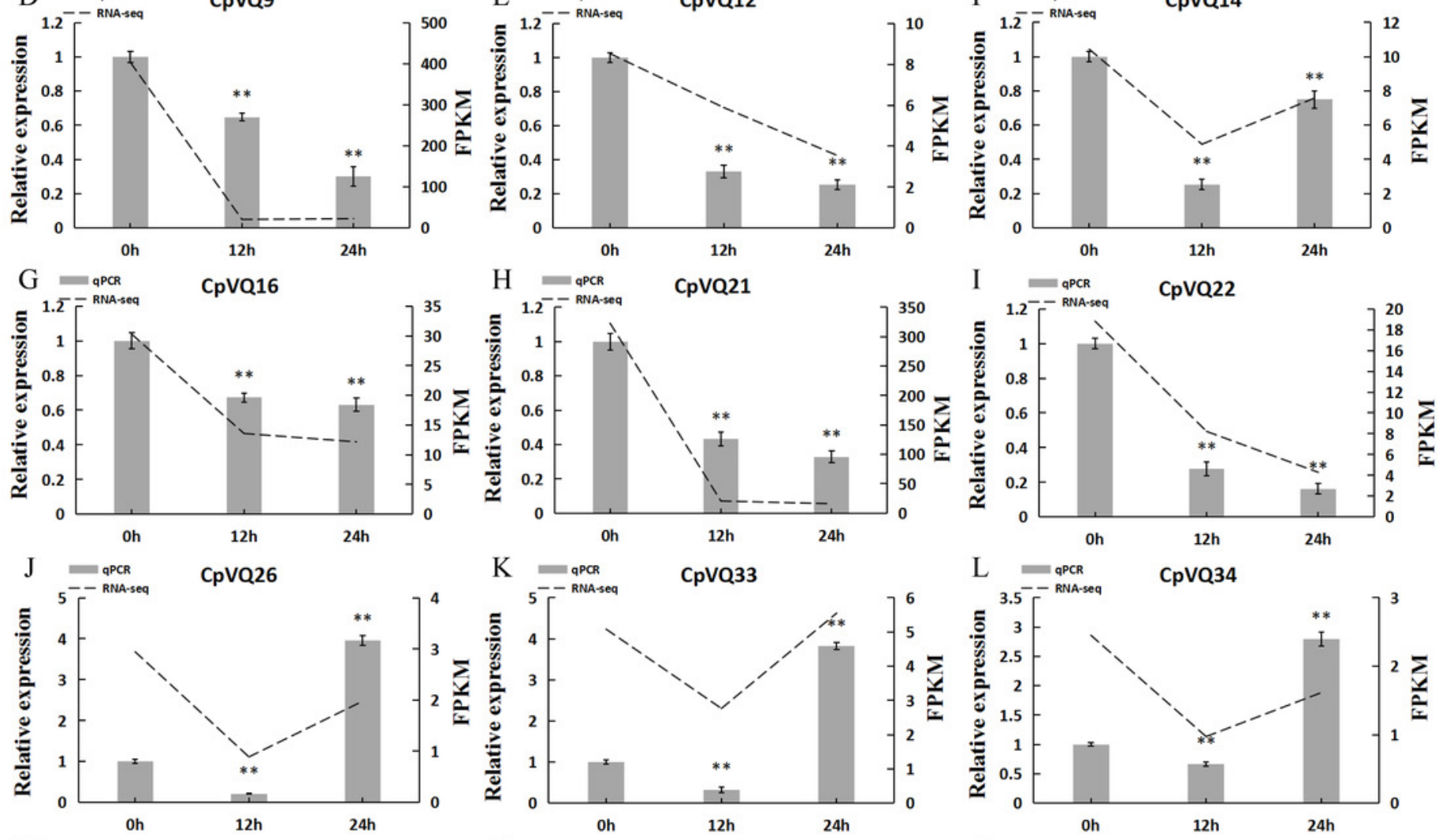

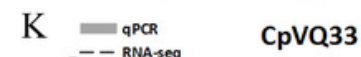

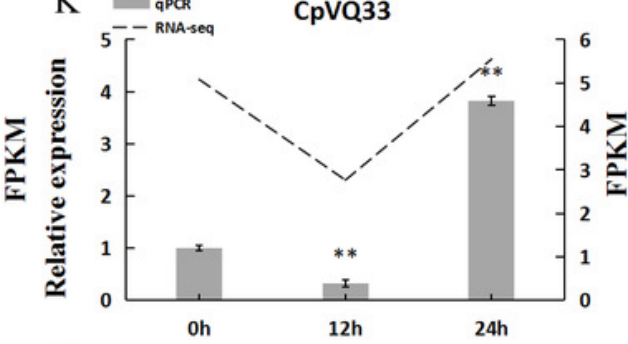

$\mathrm{L}=$ accR $\quad$ CPVQ34
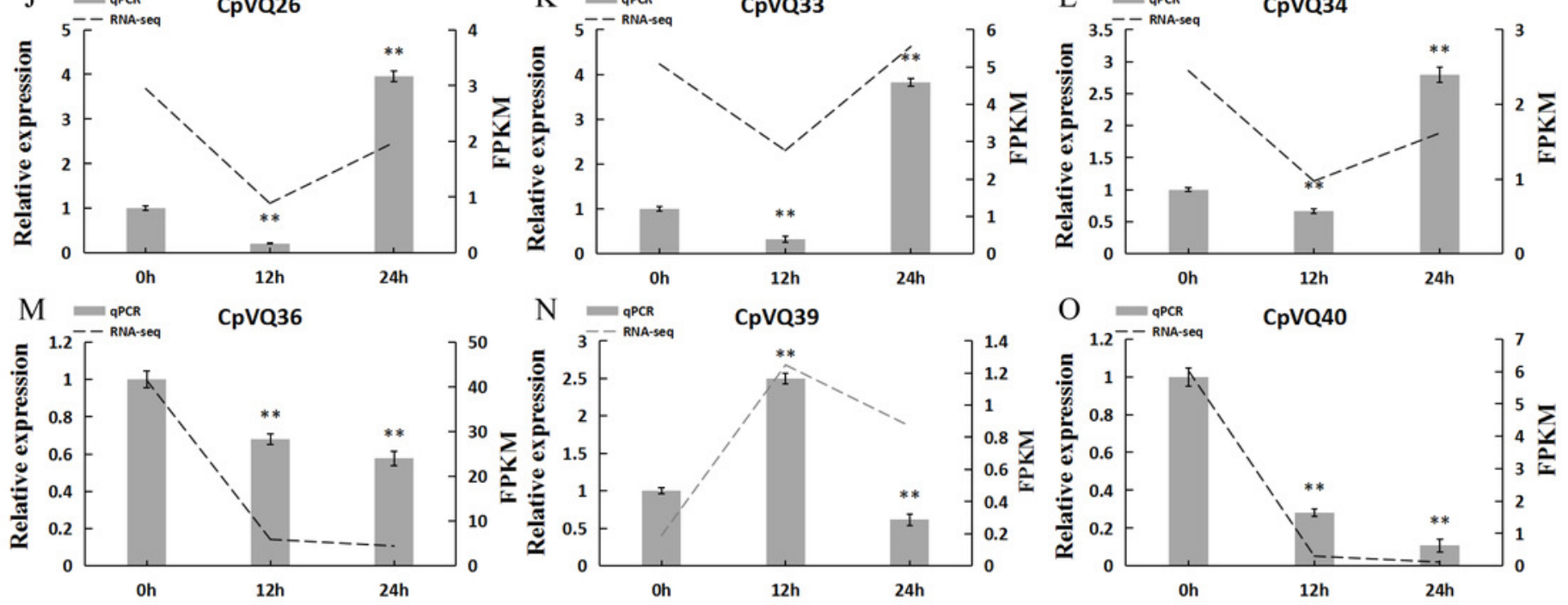
Figure 9

qRT-PCR validation of VQ genes in the response to drought treatment.

Stress treatments and time course are described in "Materials \& Methods". (A-O) represent different genes that were evaluated by qRT-PCR. Asterisks indicate statistically significant differences between the stressed samples and counterpart controls $(* p<0.05, * * p<0.01)$. 


\section{Drought}
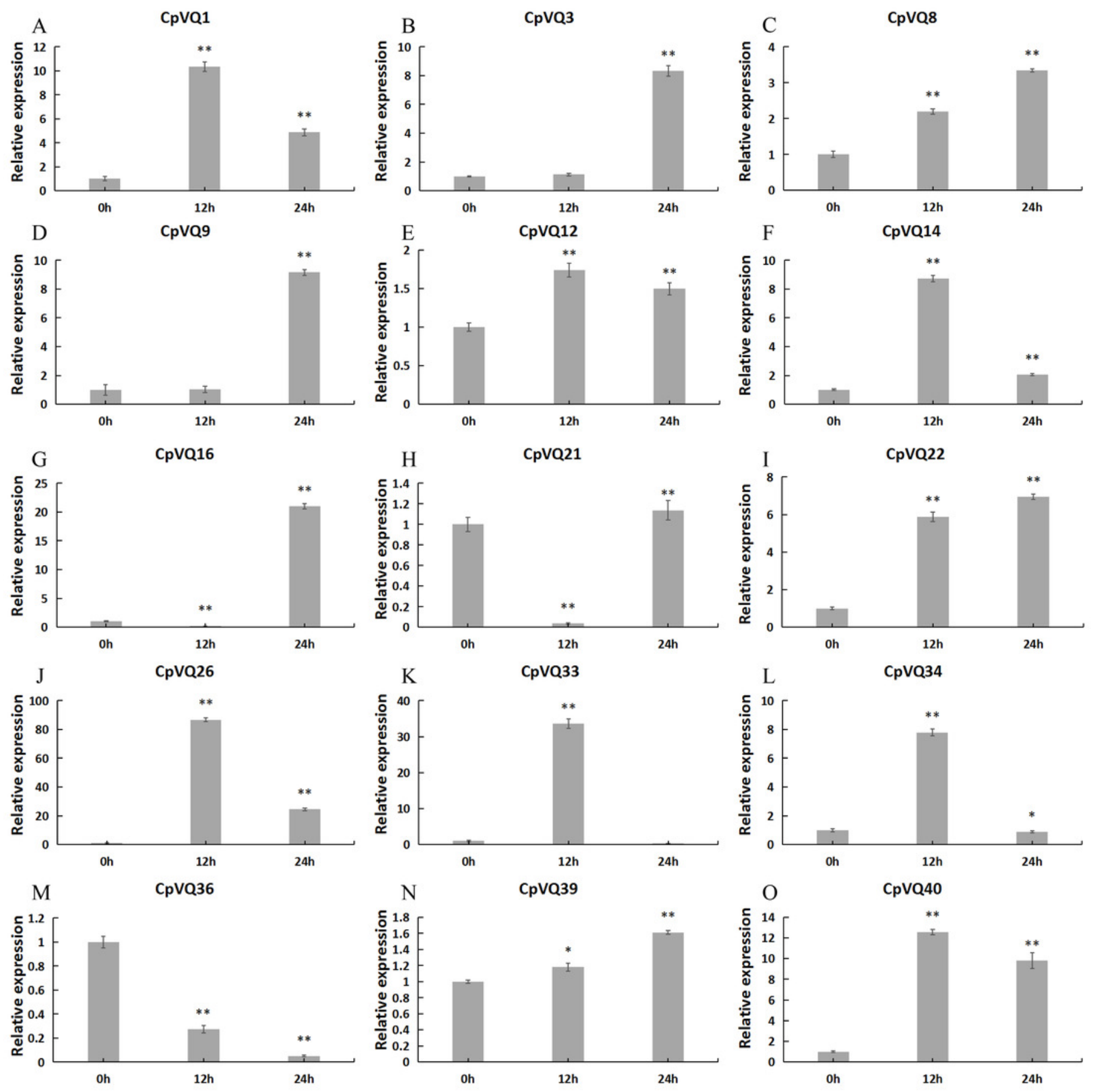


\section{Figure 10}

qRT-PCR validation of VQ genes in the response to cold treatment.

Stress treatments and time course are described in "Materials \& Methods". (A-O) represent different genes that were evaluated by qRT-PCR. Asterisks indicate statistically significant differences between the stressed samples and counterpart controls $(* p<0.05, * * p<0.01)$. 
Cold
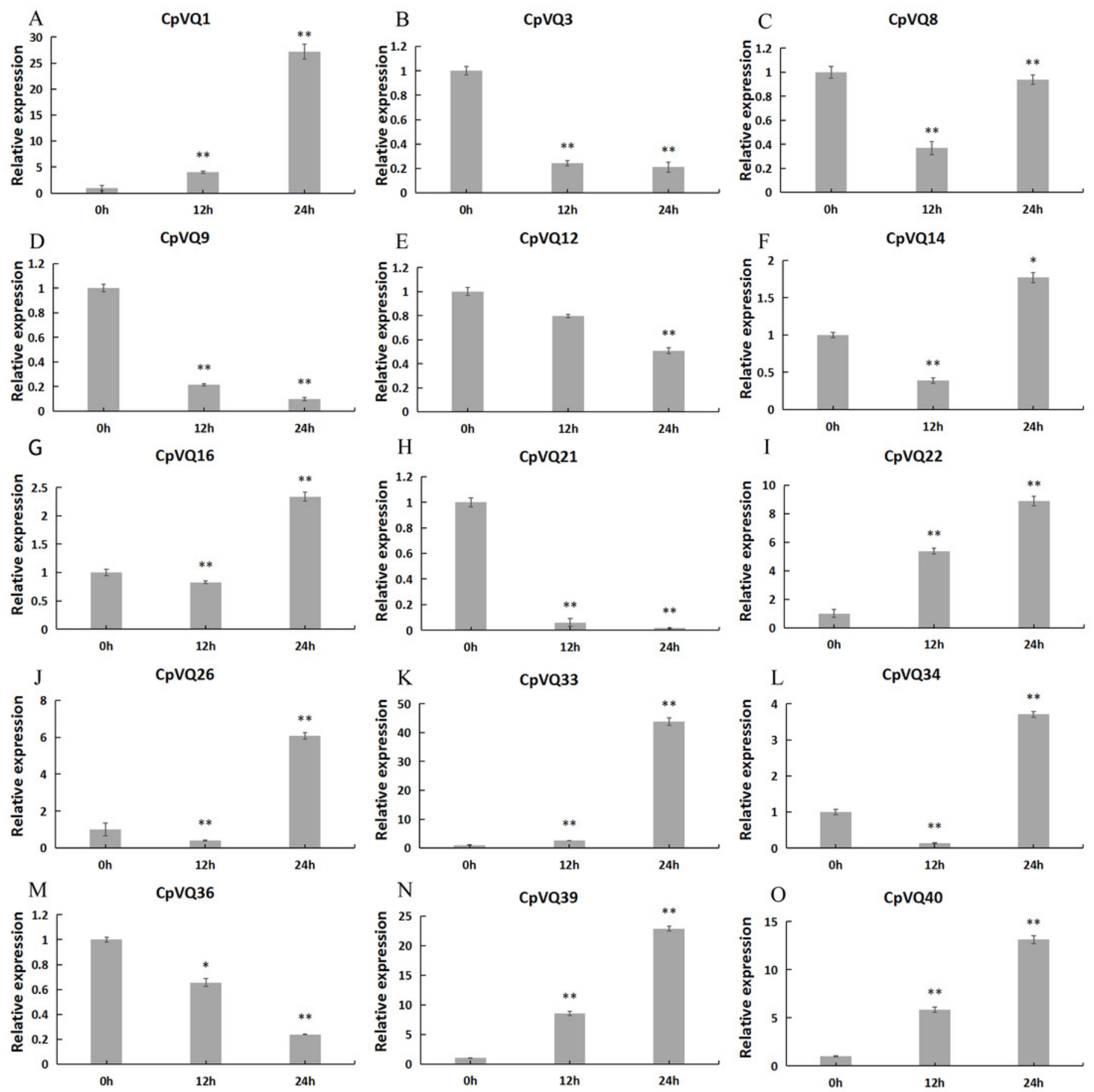
Figure 11

qRT-PCR validation of $V Q$ genes in the response to salt treatment.

Stress treatments and time course are described in "Materials \& Methods". (A-O) represent different genes that were evaluated by qRT-PCR. Asterisks indicate statistically significant differences between the stressed samples and counterpart controls $(* p<0.05, * * p<0.01)$. 
Salt
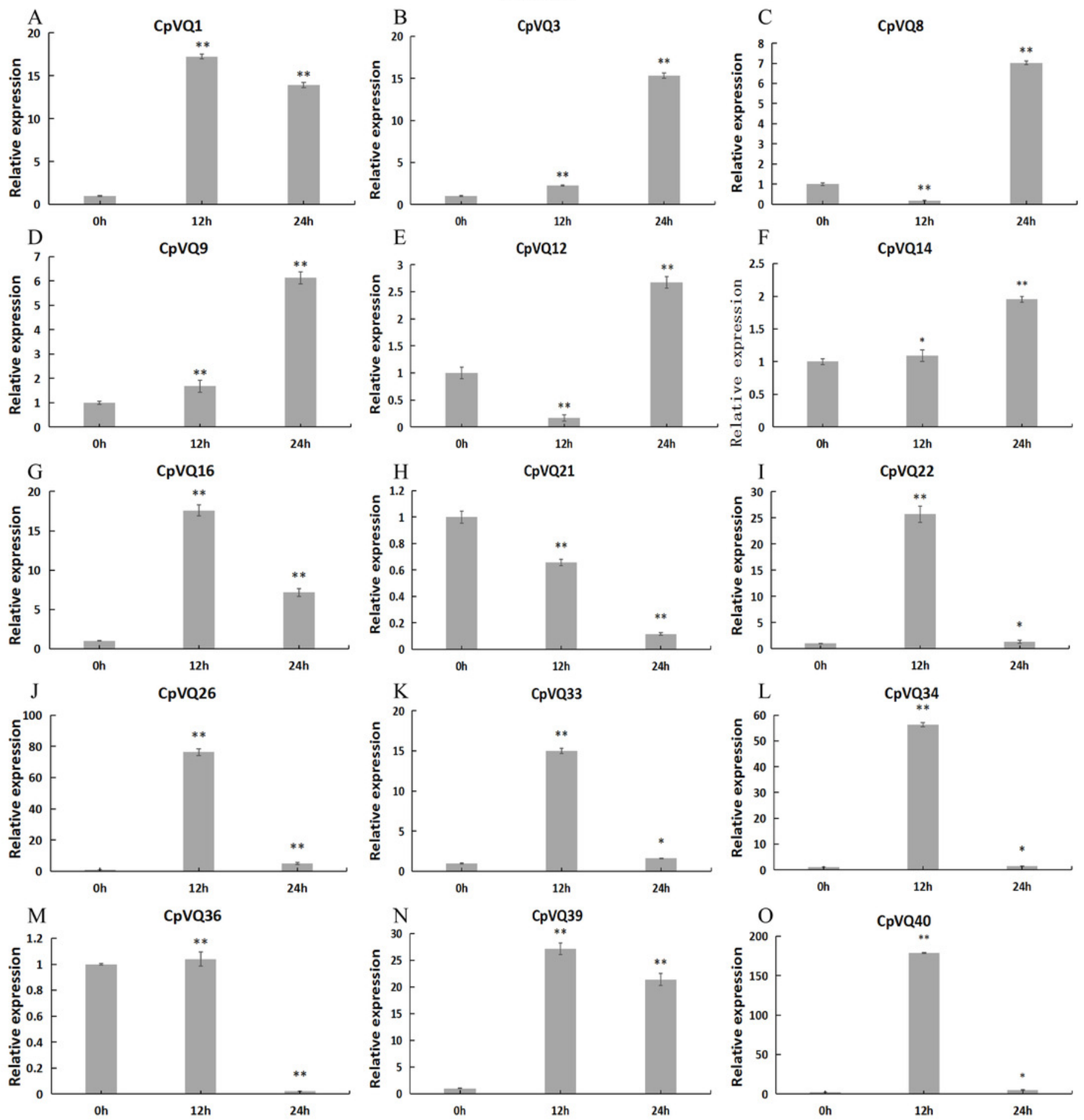


\section{Figure 12}

qRT-PCR validation of $V Q$ genes in the response to waterlogging treatment.

Stress treatments and time course are described in "Materials \& Methods". (A-O) represent different genes that were evaluated by qRT-PCR. Asterisks indicate statistically significant differences between the stressed samples and counterpart controls $(* p<0.05, * * p<0.01)$. 

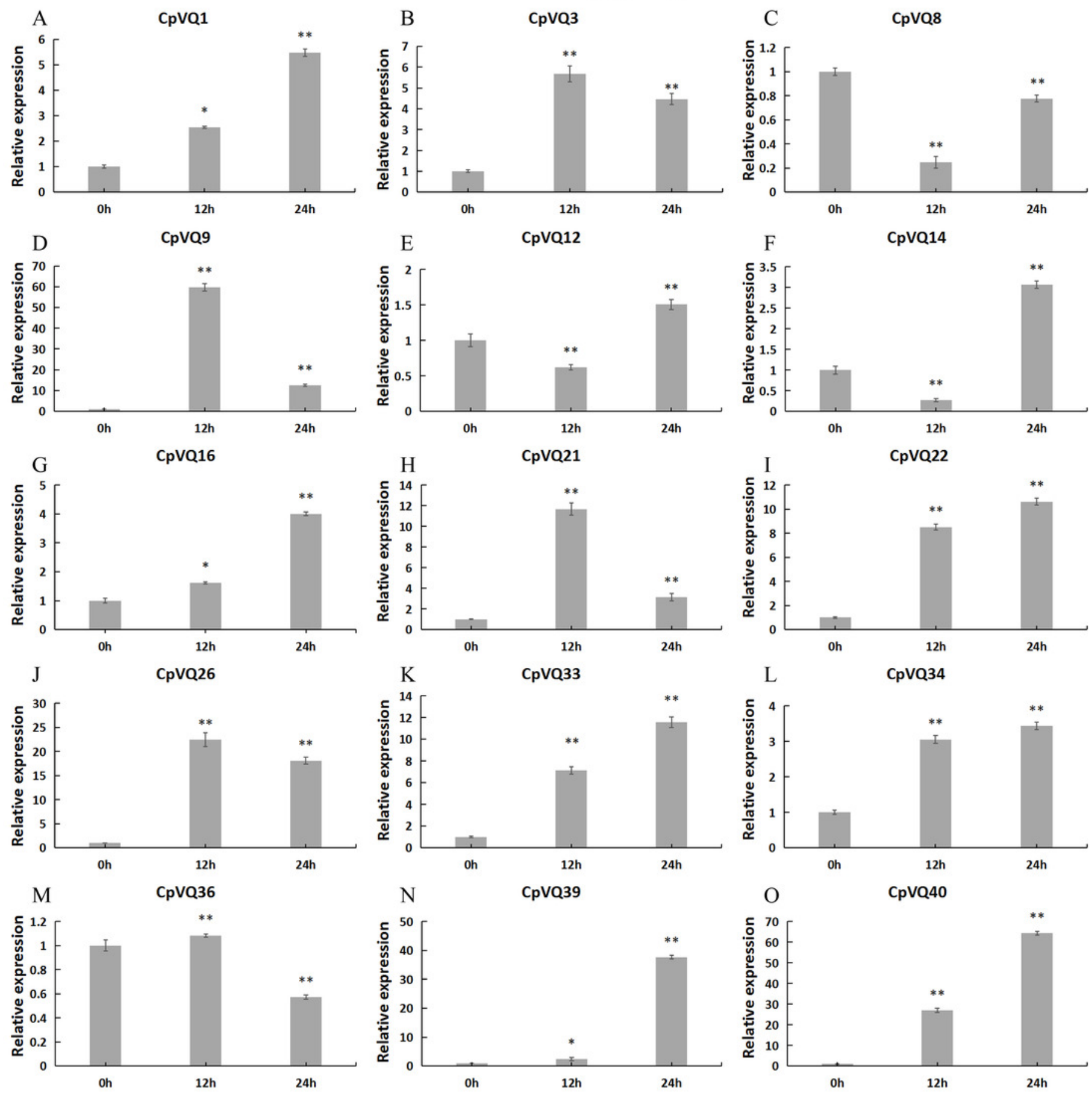
Table $\mathbf{1}$ (on next page)

List of all VQ genes identified in C.pepo 


\section{Table 1}

\section{$2 \quad$ List of all VQ genes identified in Cucurbita pepo $L$.}

\begin{tabular}{|c|c|c|c|c|c|c|}
\hline $\begin{array}{l}\text { Gene } \\
\text { name }\end{array}$ & Gene locus & $\begin{array}{c}\text { Chromosome } \\
\text { location }\end{array}$ & $\begin{array}{l}\text { Length } \\
\text { (aa) }\end{array}$ & $\mathrm{pI}$ & $\begin{array}{l}\text { Molecular } \\
\text { weight } \\
\text { (KDa) }\end{array}$ & $\begin{array}{l}\text { Family } \\
\text { group }\end{array}$ \\
\hline CpVQ1 & Cp4. 1LG00g04520 & 16693604-16694523 & 160 & 5.44 & 17313.57 & III \\
\hline CpVQ2 & Cp4. 1LG00g15310 & $42412302-42412658$ & 118 & 9.83 & 13171.89 & II \\
\hline CpVQ3 & Cp4. 1LG01g01160 & LG01: $3157885-3158544$ & 219 & 9.03 & 22938.39 & $\mathrm{~V}$ \\
\hline CpVQ4 & Cp4. 1LG01g09330 & LG01: $4248892-4250868$ & 389 & 6.8 & 41553.79 & V \\
\hline CpVQ5 & Cp4. 1LG01g21070 & $\begin{array}{l}\text { LG01: } 17809037- \\
17809507\end{array}$ & 156 & 8.13 & 17422.62 & II \\
\hline CpVQ6 & Cp4. 1LG02g02700 & LG02: $3577192-3577794$ & 200 & 6.91 & 21690.73 & $\mathrm{~V}$ \\
\hline CpVQ7 & Cp4. 1LG02g03510 & LG02: $3102280-3103518$ & 283 & 9. 16 & 30711.29 & V \\
\hline CpVQ8 & Cp4. 1LG02g05130 & LG02: 2005092-2009226 & 118 & 9.83 & 13178.19 & II \\
\hline CpVQ9 & Cp4. 1LG03g04140 & LG03: $2346972-2347676$ & 234 & 6.53 & 25197.91 & VI \\
\hline CpVQ10 & Cp4. 1LG04g14070 & $\begin{array}{l}\text { LG04: } 11310784- \\
11311509\end{array}$ & 241 & 5.94 & 25762.45 & II \\
\hline CpVQ11 & Cp4. 1LG05g10550 & LG05: $7053658-7054299$ & 213 & 6.51 & 22984.1 & II \\
\hline CpVQ12 & Cp4. 1LG06g02740 & LG06: $1556739-1557260$ & 173 & 10.13 & 18950.64 & IV \\
\hline CpVQ13 & Cp4. 1LG06g04260 & LG06: $2472160-2473164$ & 334 & 9.91 & 35686.43 & VII \\
\hline CpVQ14 & Cp4. 1LG06g05110 & LG06: 3011929-3017926 & 628 & 5.57 & 67834.1 & $\mathrm{~V}$ \\
\hline CpVQ15 & Cp4. 1LG06g05610 & LG06: $3325430-3326068$ & 113 & 9.62 & 12485.03 & $\mathrm{~V}$ \\
\hline CpVQ16 & Cp4. 1LG06g06820 & LG06: $4263922-4264659$ & 245 & 8.71 & 26210.14 & IV \\
\hline CpVQ17 & Cp4. 1LG07g01470 & LG07: 802433-803374 & 313 & 10.08 & 33653.95 & VII \\
\hline CpVQ18 & Cp4. 1LG07g09800 & LG07: $8797116-8797493$ & 125 & 6.05 & 14220.9 & III \\
\hline CpVQ19 & Cp4. 1LG08g09380 & LG08: 7407091-7407423 & 110 & 10.67 & 12069.01 & II \\
\hline CpVQ20 & Cp4. 1LG08g13390 & LG08: $9672254-9672643$ & 129 & 4.84 & 14612.33 & III \\
\hline CpVQ21 & Cp4. 1LG08g13490 & LG08: 9735143-9735811 & 222 & 5.92 & 23933.64 & VI \\
\hline CpVQ22 & Cp4. 1LG09g08360 & LG09: $7719957-7720586$ & 209 & 9.64 & 22441.4 & IV \\
\hline CpVQ23 & Cp4. 1LG10g01130 & LG10: 3319719-3322117 & 425 & 8.71 & 45497.21 & $\mathrm{~V}$ \\
\hline CpVQ24 & Cp4. 1LG10g01930 & LG10: $2886496-2886996$ & 166 & 9.23 & 18288.05 & VIII \\
\hline CpVQ25 & Cp4. 1LG10g04880 & LG10: 1115416-1116003 & 195 & 9.05 & 21186.84 & IV \\
\hline CpVQ26 & Cp4. 1LG10g06710 & LG10: 166830-167354 & 174 & 6.28 & 18860.78 & VI \\
\hline CpVQ27 & Cp4. 1LG10g11090 & LG10: $7510307-7511283$ & 164 & 5.72 & 17629.89 & III \\
\hline CpVQ28 & Cp4. 1LG10g12290 & LG10: $9255696-9256184$ & 162 & 10.05 & 18042.53 & IV \\
\hline CpVQ29 & Cp4. 1LG11g04560 & LG11: $2540172-2540996$ & 274 & 10.43 & 30231.7 & VII \\
\hline
\end{tabular}




\begin{tabular}{|c|c|c|c|c|c|c|}
\hline CpVQ30 & Cp4. 1LG12g07320 & LG12: 6924419-6924844 & 141 & 5.91 & 15294. 24 & IV \\
\hline CpVQ31 & Cp4. 1LG13g04200 & LG13: 6294724-6297911 & 282 & 9. 99 & 31079.58 & II \\
\hline CpVQ32 & Cp4. 1LG14g01330 & LG14: $3722302-3723387$ & 425 & 8. 71 & 45497.21 & $\mathrm{~V}$ \\
\hline CpVQ33 & Cp4. 1LG14g03000 & LG14: $2622622-2623233$ & 203 & 9.68 & 21226.55 & $\mathrm{~V}$ \\
\hline CpVQ34 & Cp4. 1LG14g03270 & LG14: $2365261-2365482$ & 73 & 9.15 & 8344.47 & I \\
\hline CpVQ35 & Cp4. 1LG16g00810 & LG16: 1567379-1568005 & 208 & 6.3 & 22405.61 & II \\
\hline CpVQ36 & Cp4. 1LG17g08680 & LG17: $5075487-5075909$ & 140 & 7.84 & 14816.74 & IV \\
\hline CpVQ37 & Cp4. 1LG19g00160 & LG19: 110455-110994 & 179 & 10. 04 & 19803.85 & IV \\
\hline CpVQ38 & Cp4. 1LG19g02600 & LG19: $2163376-2163876$ & 166 & 4.93 & 17768.9 & III \\
\hline CpVQ39 & Cp4. 1LG19g02610 & LG19: $2160888-2161388$ & 166 & 4.93 & 17768.9 & III \\
\hline CpVQ40 & Cp4. 1LG19g06070 & LG19: $7655240-7655563$ & 107 & 4. 68 & 12049.37 & I \\
\hline CpVQ41 & Cp4. 1LG19g07250 & LG19: 7111871-7112545 & 224 & 9.62 & 24493. 82 & IV \\
\hline CpVQ42 & Cp4. 1LG19g09780 & LG19: $5873440-5875876$ & 344 & 6.26 & 36896.06 & $\mathrm{~V}$ \\
\hline CpVQ43 & Cp4. 1LG19g10580 & LG19: $5411418-5411909$ & 163 & 9.66 & 17828.64 & VIII \\
\hline CpVQ44 & Cp4. 1LG20g04410 & LG20: $2519217-2520794$ & 335 & 8.85 & 36902.06 & IV \\
\hline
\end{tabular}




\section{Table 2 (on next page)}

$\mathrm{Ka}$, Ks and Ka/Ks values calculated for homologous VQ gene pairs. 


\section{Table2}

$2 \mathrm{Ka}, \mathrm{Ks}$ and $\mathrm{Ka} / \mathrm{Ks}$ values calculated for homologous VQ gene pairs.

\begin{tabular}{|c|c|c|c|c|c|}
\hline Gene1 & Gene2 & $\mathbf{K a}$ & $\mathbf{K s}$ & $\begin{array}{c}\mathrm{Ka} / \mathrm{Ks} \\
\text { ratio }\end{array}$ & $\begin{array}{c}\text { Differentiation } \\
\text { time }\end{array}$ \\
\hline CpVQ3 & CpVQ33 & 0.143408874 & 0.41714233 & 0.343788832 & 34.19199426 \\
\hline CpVQ4 & CpVQ23 & 0.56179148 & 1.733083058 & 0.324157274 & 142.0559883 \\
\hline CpVQ4 & CpVQ32 & 0.114789648 & 0.572645983 & 0.200454821 & 46.93819533 \\
\hline CpVQ5 & CpVQ31 & 0.244725293 & 0.735263756 & 0.332840142 & 60.26752102 \\
\hline CpVQ6 & CpVQ15 & 1.018237411 & 1.77385189 & 0.574026172 & 145.3976959 \\
\hline CpVQ8 & CpVQ13 & 0.074887932 & 0.430075478 & 0.174127416 & 35.25208839 \\
\hline CpVQ8 & CpVQ17 & 0.302766804 & 1.921171796 & 0.157594862 & 157.473098 \\
\hline CpVQ8 & CpVQ29 & 0.319999352 & 1.905949455 & 0.167894983 & 156.2253652 \\
\hline CpVQ9 & CpVQ21 & 0.895462518 & $\mathrm{NaN}$ & $\mathrm{NaN}$ & \#VALUE! \\
\hline CpVQ11 & CpVQ35 & 0.083226176 & 0.468993438 & 0.177457016 & 38.4420851 \\
\hline CpVQ13 & CpVQ17 & 0.304326774 & 1.957821306 & 0.155441548 & 160.4771563 \\
\hline CpVQ13 & CpVQ29 & 0.301640689 & 2.424748395 & 0.12440082 & 198.7498684 \\
\hline CpVQ16 & CpVQ22 & 0.351584842 & 2.560617395 & 0.137304715 & 209.8866717 \\
\hline CpVQ22 & CpVQ25 & 0.193161647 & 1.846190406 & 0.104627153 & 151.3270824 \\
\hline CpVQ22 & CpVQ41 & 0.206138134 & 1.465932276 & 0.140619139 & 120.1583833 \\
\hline CpVQ23 & CpVQ32 & 0.614828236 & 2.638504545 & 0.233021481 & 216.2708644 \\
\hline
\end{tabular}


$\begin{array}{llllll}\text { CpVQ23 } & \text { CpVQ42 } & 0.202547017 & 0.536664673 & 0.377418203 & 43.98890764\end{array}$

$\begin{array}{llllll}\text { CpVQ24 } & \text { CpVQ43 } & 0.142828118 & 0.572200927 & 0.249611826 & 46.90171536\end{array}$

$\begin{array}{llllll}\text { CpVQ25 } & \text { CpVQ41 } & 0.111174117 & 0.440146678 & 0.252584246 & 36.07759653\end{array}$

$\begin{array}{llllll}\text { CpVQ27 } & \text { CpVQ39 } & 0.182897122 & 0.455285224 & 0.401719872 & 37.31846099\end{array}$

$\begin{array}{llllll}\text { CpVQ30 } & \text { CpVQ36 } & 0.207558725 & 0.385917729 & 0.537831536 & 31.6326007\end{array}$

3 